\title{
Heavy ion accelerator facility front end design and commissioning
}

\author{
Y. Yang ${ }^{*}$ L. T. Sun, Y. H. Zhai, Y. J. Zhai, Z. Shen, C. Qian, W. P. Dou, W. Ma, L. Lu, \\ Y. H. Guo, X. Fang, Y. J. Yuan, L. P. Sun, J. C. Yang, and H. W. Zhao ${ }^{\dagger}$ \\ Institute of Modern Physics, Chinese Academy of Sciences, Lanzhou, 730000, China
}

(Received 5 May 2019; published 27 November 2019)

\begin{abstract}
A new project, high intensity heavy ion accelerator facility (HIAF), is currently under design and construction in China. The HIAF front end, composed of electron cyclotron resonance (ECR) ion sources, low energy beam transport (LEBT) and radio frequency quadrupole (RFQ), will produce and provide beams of ions with a mass up to uranium at a beam energy of $0.5 \mathrm{MeV} / \mathrm{u}$. The typical beam intensity is designed up to $2 \mathrm{emA}$ for the uranium beam with a charge state of $35+$. This paper presents an overall design of the front end for HIAF and discusses several key issues in the design. By modeling the beam extraction from the ECR source, we got a reliable starting beam condition to perform the design. Transverse coupling of the beam from the source was elaborated. To relieve the coupling we implanted two solenoids after the source. Space charge effect in the charge state selection of the ion source was evaluated. An overall space charge compensation degree of no less than $70 \%$ was predicted. A beam dynamics simulation was performed by using the initial particle distribution obtained from the extraction modeling. The simulation resulted in development of a beam collimation system in the LEBT to confine the transverse emittance. The RFQ design will follow the development of LEAF-RFQ at Institute of Modern Physics, which has successfully commissioned with several beams and demonstrated as an excellent design. Recent beam commissioning results of LEAF-RFQ will also be presented in this paper.
\end{abstract}

DOI: $10.1103 /$ PhysRevAccelBeams.22.110101

\section{INTRODUCTION}

As shown in Fig. 1, the heavy ion accelerator facility (HIAF) project $[1,2]$ consists of ion sources, linear accelerator, synchrotrons and several experimental terminals. The superconducting electron cyclotron resonance (ECR) source is used to provide highly charged ion beams, and a $2.45 \mathrm{GHz}$ proton source is used to provide $\mathrm{H}_{3}{ }^{+}$beam. The superconducting ion linac accelerator (iLinac) is designed to accelerate ions with the charge-mass ratio $Q / A=1 / 6.8$ (e.g., ${ }^{238} \mathrm{U}^{35}$ ) to the energy of $17 \mathrm{MeV} / \mathrm{u}$. Ions provided by iLinac will be cooled, accumulated and accelerated to the required intensity and energy (up to $1 \times 10^{11} \mathrm{ppp}$ and $800 \mathrm{MeV} / \mathrm{u}$ of ${ }^{238} \mathrm{U}^{35}{ }^{+}$) in the booster ring (BRing), then fast extracted and transferred either to the external targets or the spectrometer ring (SRing). It is also planned to equip BRing with a slow extraction system for a wide range of applied research in biology and material science. As a key part of the HIAF complex, SRing is designed as a

\footnotetext{
*Corresponding author. yangyao@impcas.ac.cn

zhaohw@impcas.ac.cn

Published by the American Physical Society under the terms of the Creative Commons Attribution 4.0 International license. Further distribution of this work must maintain attribution to the author(s) and the published article's title, journal citation, and DOI.
}

multifunction experimental storage ring. A time-of-flight detector system will be installed for nuclei mass measurements with isochronous mode. Highly purified radioactive beams can be extracted from SRing for nuclear physics experiments.

The iLinac could have three operation modes. The first is to use the iLinac as an independent machine, operating at $\mathrm{cw}$ mode and providing $\mathrm{cw}$ beams to the iLinac terminal for experiments. The second mode is to use the iLinac only for injection into BRing. Since BRing requires pulsed beam with frequency from 0.3 to $5 \mathrm{~Hz}$ and pulse width from 0.2 to $2 \mathrm{~ms}$, iLinac would also operate with pulsed mode. In this mode, the ECR source could work at afterglow mode to produce much higher peak currents, such as $2 \mathrm{emA}^{209} \mathrm{Bi}^{31+}$ and $2 \mathrm{emA}^{238} \mathrm{U}^{35^{+}}$. The third mode is called parallel mode, meaning iLinac would accelerate two beams simultaneously with one beam extracted to the experimental terminal of iLinac, and the other injected to BRing. Two ion sources could provide beams with similar charge to mass ratios simultaneously. Beam switching and time structure modulation could be operated by the low energy beam transport (LEBT) lines.

This paper focuses on the room-temperature front end of HIAF and presents an overall design. Key base and issues in the design, especially for the intense heavy ion beam transmission and acceleration, were discussed. The paper mainly consists of three parts. In the first part, we will 


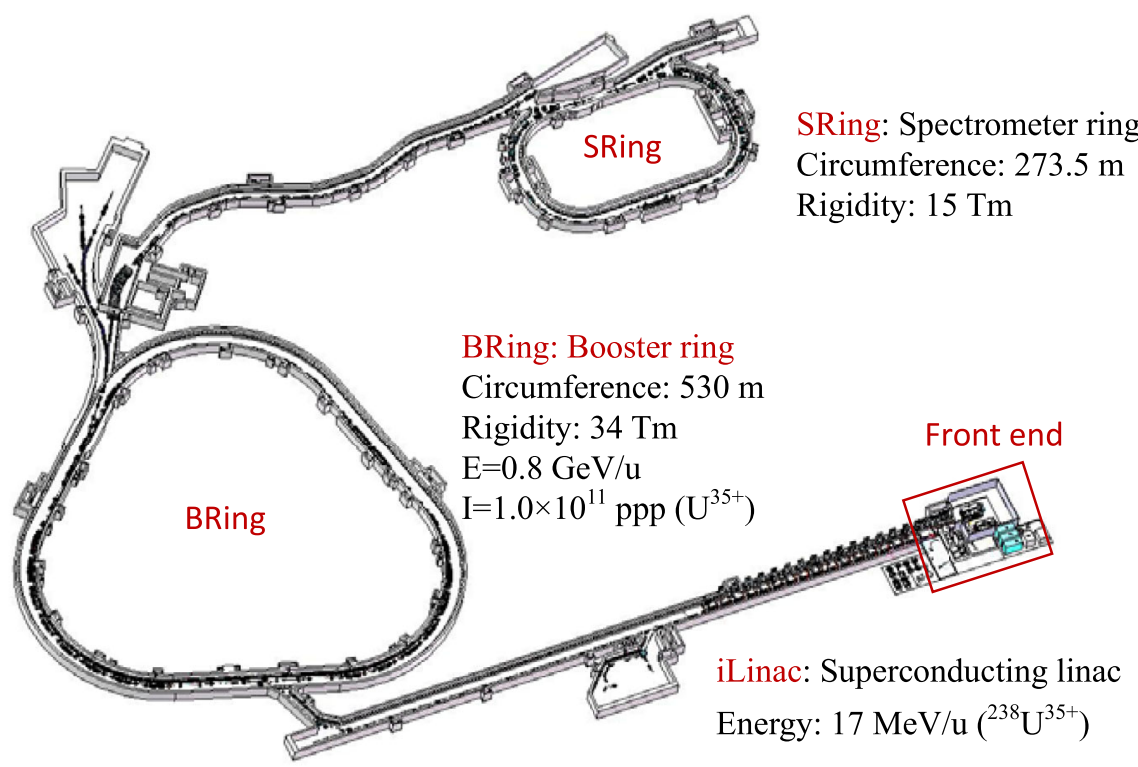

FIG. 1. An overview of the HIAF complex.

discuss the ion beam extraction from the ECR source and multispecies beam analyzing. The second part is focused on the beam transmission in the LEBT, and the third part is about the radio frequency quadrupole (RFQ) design and beam commissioning.

\section{HIAF FRONT END}

The HIAF front end includes three ion sources, LEBT lines and a cw RFQ, as shown in Fig. 2. SECRAL [3], one of the best performing third generation superconducting ECR sources, can be one of the operational ion sources for
HIAF operation. $45 \mathrm{GHz}$ FECR [4] (first fourth generation ECR ion source), which is being developed at Institute of Modern Physics (IMP), will meet the HIAF requirement for high intensity of those heavy ion beams. A $2.45 \mathrm{GHz}$ proton source can be employed to supply $\mathrm{H}_{3}{ }^{+}$beam. The main goal of the LEBT line is to separate efficiently the different masses and charge states coming from the source and deliver the selected beam to the RFQ. Each ion source has its associated LEBT line and the lines merge into one connecting with the RFQ. A similar structure can be found in Spiral-2 [5] at GANIL and FRIB [6] at MSU. After each charge selection section of the sources, a set of diagnostic

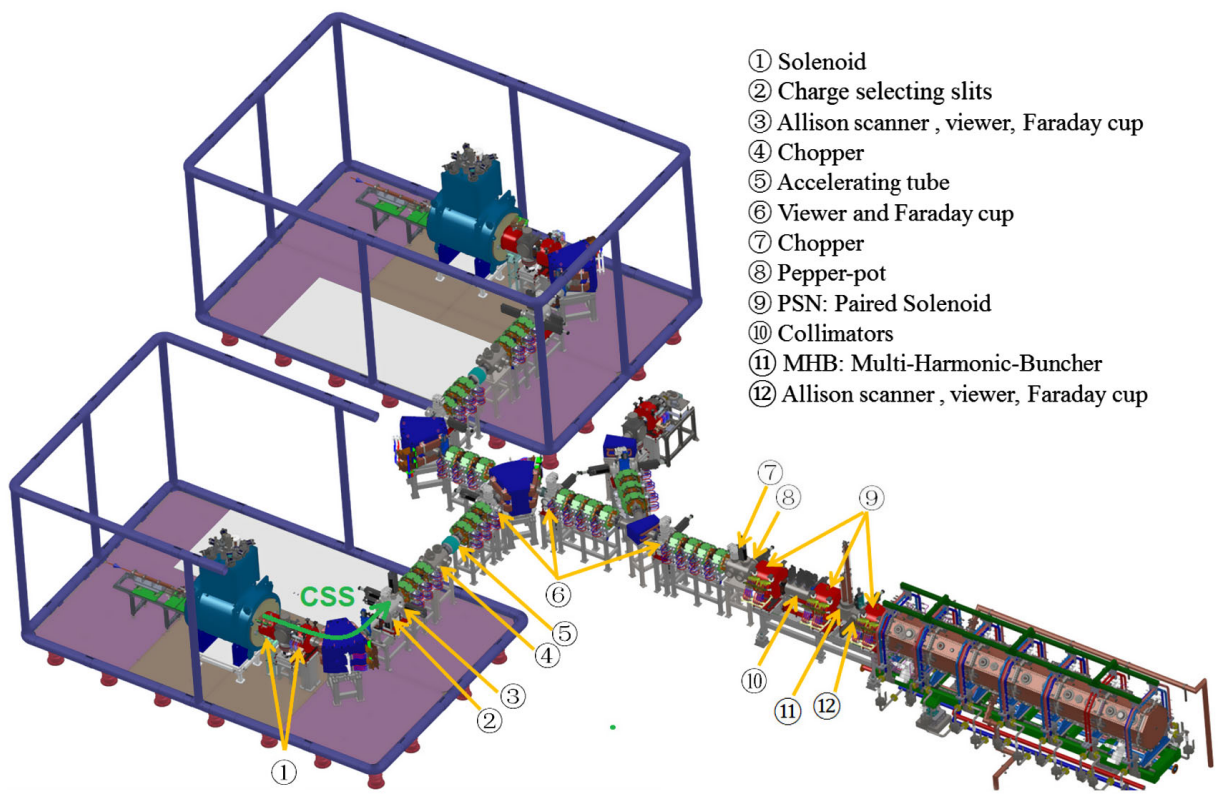

FIG. 2. HIAF Front end layout. 
devices, including two Allison-type emittance meters, a Faraday cup and a fluorescent target, are available. Each ion source associated line equips with an electrostatic chopper, which provides a possibility for beam switching and pulse length controlling while the iLinac operates at parallel mode. The LEBT systems are achromatic, designed to minimize the horizontal emittance growth induced by the beam energy spread from the source while transporting through the analyzing magnets. Two $90^{\circ}$ dipoles are used with six magnetic quadrupoles between them to build an achromat and control the beam size. Due to the required beam energy of $14 \mathrm{keV} / \mathrm{u}$ by the RFQ, heavy ion ${ }^{238} \mathrm{U}^{35^{+}}$, for example, needs to be accelerated with a $95.2 \mathrm{kV}$ high voltage (HV). However, the extraction voltage of the ion source is typically below $50 \mathrm{kV}$ limited by sparking risk, therefore two $100 \mathrm{kV}$ accelerating tubes will be employed in the SECRAL and FECR associated lines respectively to reach the required energy of the RFQ and all upstream elements will be put on the HV platforms. Another chopper is used before RFQ especially for quick machine protection. To reduce beam losses in the RFQ and superconducting linac, the beam is collimated by three apertures in the LEBT. Before the collimation channel a pepper-pot emittance meter [7] is located for fast emittance measurement. The direct-current beam is bunched before injection into the RFQ by a multiharmonic buncher (MHB). Another set of diagnostic devices (two Allison scanners, a Faraday cup and a fluorescent target) are placed just after the MHB and share a chamber with the MHB. The transverse focusing element before the RFQ is a so-called paired-solenoid (PSN), which is composed of two combined coils, producing opposite-polarity magnetic fields. The PSN does not give rise to a correlation between horizontal and vertical directions, avoiding emittance growth. More details about the PSN can be found in [8]. The RFQ will utilize a four-vane structure at a frequency of $81.25 \mathrm{MHz}$ to accelerate heavy ion beams with charge states charge-to-mass ratio $(Q / A)$ between $1 / 7$ and $1 / 3$ from $14 \mathrm{keV} / \mathrm{u}$ to $0.5 \mathrm{MeV} / \mathrm{u}$.

\section{ION BEAM EXTRACTION AND ANALYZING}

\section{A. Beam extraction from ECRIS}

A total current of more than $20 \mathrm{emA}$ is expected to be extracted from the superconducting ECR ion source.
Therefore, the ion source extraction voltage is designed to be biased up to $50 \mathrm{kV}$ to reduce the space charge effect. An on-line movable four-electrode system (includes a plasma electrode, a midelectrode, a suppression electrode and a grounded electrode) will be adopted so that the plasma meniscus shape and initial beam formation can be optimized by adjusting the distance between the plasma electrode and the midelectrode. Extraction simulations are necessary because it is essential for performing beam simulation to have a reasonable starting beam condition from the source. Besides, the optimization of the beam extraction itself has the potential to yield performance improvement. IBSimu [9] code was used to model the extraction of FECR since it provides good capabilities to simulate multispecies extraction from plasma in the presence of strong magnetic fields and space charge, conditions which are closely associated with ECR ion sources. Although the nonlinear positive plasma model used by the code considerably simplifies the complex ECR ion source plasma conditions, previous studies have shown that IBSimu is a powerful tool in modeling ECR ion source extraction systems [10,11].

Simulations were conducted for uranium beam extraction from FECR. The initial ion species were assumed to have a similar distribution with the measured charge state distribution (CSD, presented in [12]) for the recorded uranium beam intensity production with VENUS at LBNL [13]. However, beam currents of each ion species were scaled up by creating total extracted beams of $20 \mathrm{emA}$. Ions are extracted from plasma that is modeled in a reduced volume with assumed plasma potential of $20 \mathrm{~V}$, and a low $10 \mathrm{eV}$ temperature for the cold background electron population. A cold ion population was assumed with longitudinal and transverse temperatures of $1 \mathrm{eV}$ based on the generally accepted order of magnitude in ECR plasmas $[10,11]$. The simulated beam particles were generated inside the plasma volume with uniform radial distribution. The 3D magnetic field map of FECR, including the solenoid and hexapole fields, was calculated with OPERA 3D software and included in the simulation.

Simulated particle trajectory density of multispecies ion beam through the ion source extraction region is presented in Fig. 3. In this simulation, the voltages applied to the electrodes were set as $50,23,-2 \mathrm{kV}$ and 0 , respectively. The plasma meniscus has a flat shape when the distance between the plasma electrode and midelectrode is $27.7 \mathrm{~mm}$,

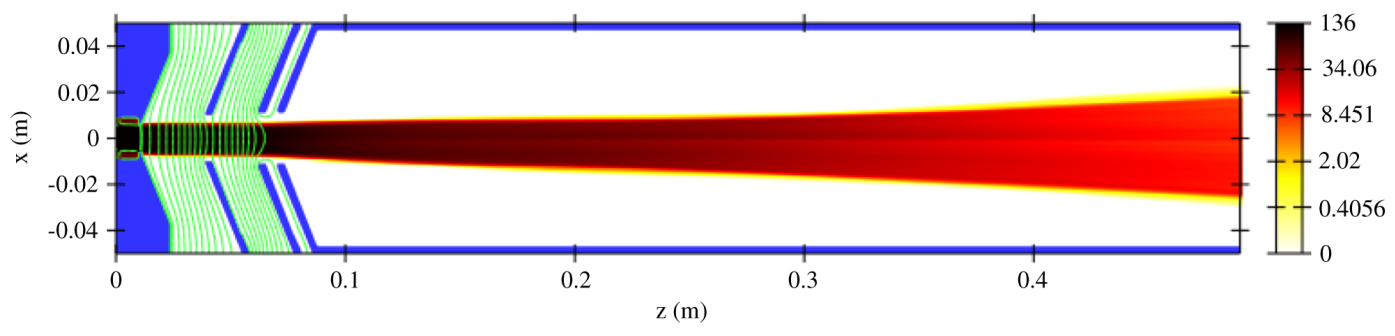

FIG. 3. Simulated particle trajectory density for the multispecies ion beams. 

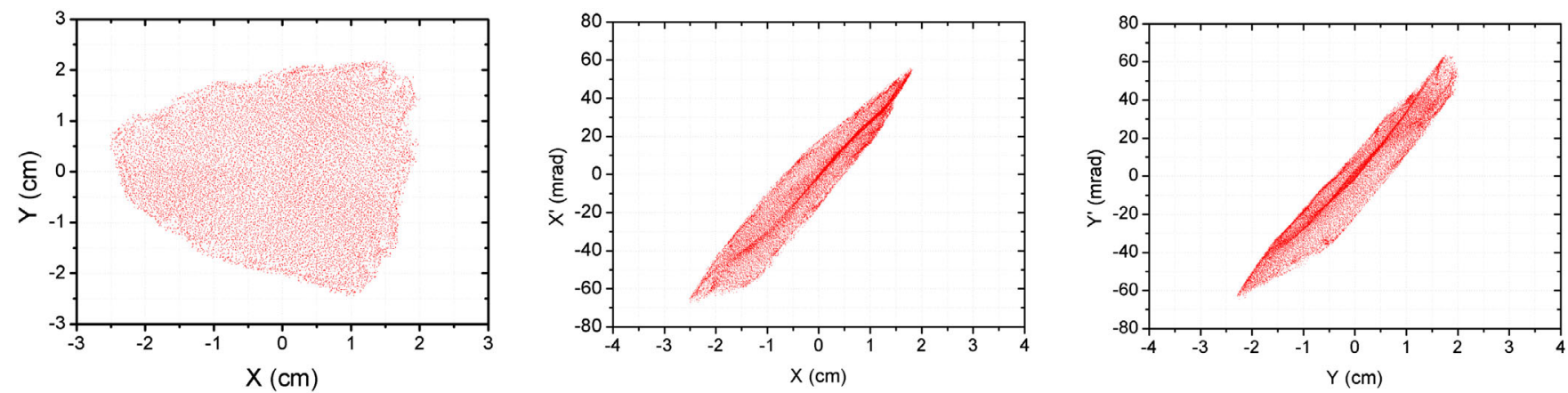

FIG. 4. $\mathrm{U}^{35+}$ beam profile in transverse plane and the phase spaces.

which indicates an initially parallel beam extraction and more uniform particle distribution in the transverse plane. The beam profile of the $\mathrm{U}^{35+}$ ion in the transverse plane and the phase spaces at the end of the extraction region (at $0.5 \mathrm{~m}$ in Fig. 3) are shown in Fig. 4. One can see the beam exhibits triangular shape and the ion density distribution in the cross section is inhomogeneous. This is the signature influence of the ECR ion source magnetic confinement structure combining sextupole and solenoid fields [14].

The ion beam is extracted from a region of high axial magnetic field, which adds a rotational component to the beam, leading to emittance growth. The magnetic contribution to the projection normalized rms emittance (in $\pi \mathrm{mm}$ mrad) [15] can be given by

$$
\varepsilon_{\mathrm{mag}}=0.032 \cdot\left(R_{\mathrm{extr}}\right)^{2} \cdot\left(\frac{B_{\mathrm{extr}}}{M / Q}\right)
$$

where $R_{\text {extr }}[\mathrm{mm}]$ is the radius of the effective extraction aperture and $M / Q$ the ion mass-to-charge ratio and $B_{\text {extr }}[\mathrm{T}]$ the maximum magnetic field at the extraction region. This equation indicates the beam emittance is proportional to the extraction field strength with the same effective extraction aperture. For FECR, $B_{\text {extr }}$ will be up to $3.5 \mathrm{~T}$ when it operates at the microwave frequency of
$45 \mathrm{GHz}$, which will induce a very large beam emittance from extraction. Calculated projection emittances for $\mathrm{U}^{35+}$ by IBSimu are of $0.32 \pi \mathrm{mm}$ mrad in both directions.

To verify the reliability of the extraction simulation, modeling for SECRAL-II [3] source extraction was conducted and a comparison between the simulation and experiment was made. As an example, Fig. 5 presents a comparison between the simulated beam profile (mixed Ar beam) and the observed beam fluorescence on a $\mathrm{KBr}$ target at the location of the extraction pumping chamber. Due to the starting conditions of the ions in the ECR plasma and overfocusing of higher charge states [10,11,14], the beams present the triangular and hollow shapes on the target. Although the simulated beam particles originate from the plasma volume with uniform radial distribution, the simulation predicts self-consistently the triangular and hollow beam structures as observed experimentally. Further comparison was made between the measured and simulated particle distributions in the phase spaces after the charge state selection (CSS, as shown in Fig. 2, from ion source exit to the charge selecting slit), illustrated in Fig. 6. $\mathrm{Ar}^{9+}$ beam was selected as the objective ion. The measurements indicated that the emittances were of $0.32 \pi \mathrm{mm}$ mrad in the horizontal and $0.26 \pi \mathrm{mm}$ mrad in the vertical, respectively. The simulations, in which the initial particle distribution
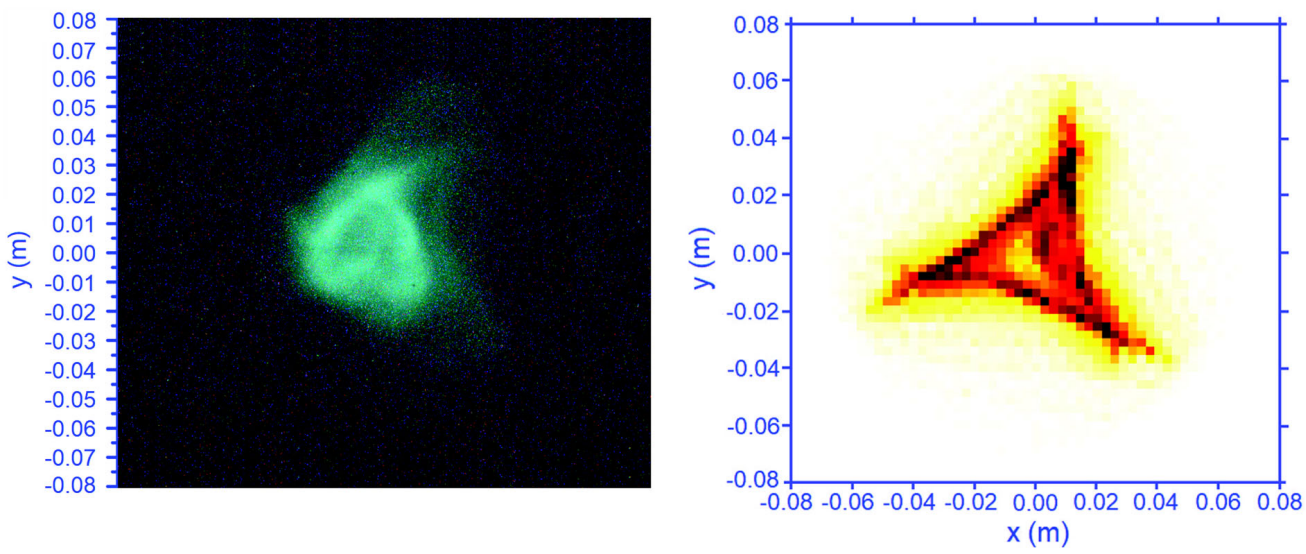

FIG. 5. Beam profile comparison between measurement (left) and simulation (right). 

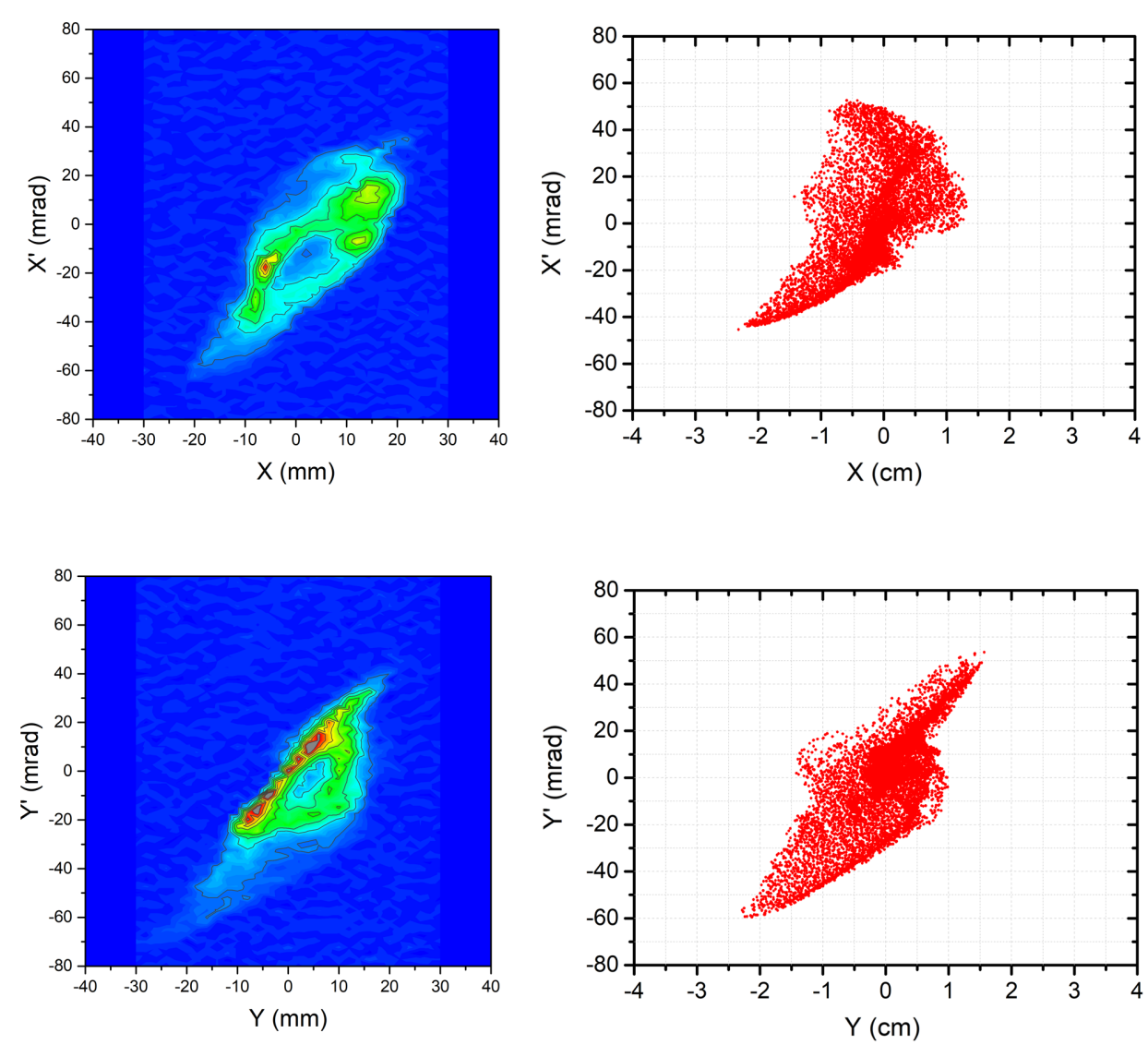

FIG. 6. Phase space comparison between measurement (left) and simulation (right).

was obtained from the extraction simulation, showed emittance of $0.31 \pi \mathrm{mm} \mathrm{mrad}$ in the horizontal and $0.27 \pi \mathrm{mm} \mathrm{mrad}$ in the vertical. Good agreements between the simulations and measurements indicate the extraction simulation could provide a reliable initial beam distribution for further beam transport simulation and beam line design.

\section{B. Transverse coupling of the beam from ECR}

Both experiments and simulations have proven the transverse coupling property of the beam from ECR ion sources [16-17]. The plasma electrode of an ECR ion source is generally located in the vicinity of a local magnetic field maximum, leading to extraction of ions from a strong magnetic field and subsequent beam formation in the descending axial magnetic field (halfsolenoid field). Two types of transverse coupling are created during beam extraction. One is due to the halfsolenoid field adding an azimuthal momentum to the beam, resulting in projection emittance growth. This type of coupling cannot be removed unless in an opposite magnetic field of the same the particles experienced while they were extracted. The second type can be attributed to the rotation effect on the beam induced by either a halfsolenoid field or a whole-solenoid field. As illustrated in the extraction simulation and observed in the experiment, ion density distribution in the cross section from the ECR source is typically inhomogeneous and presents a "non-round" beam. Therefore, beam rotation along the axis will lead to transverse coupling. This type of coupling has a periodical property and can be dissociated when the rotation angle $\Theta=n *(1 / 2 \pi)$ (where $n=0$, $\pm 1, \pm 2, \pm 3 \ldots)$ [17].

Transverse coupling can be defined by having nonzero interplane elements in the beam second moment matrix. The projection root-mean-square (rms) emittances $\varepsilon_{x}$ and $\varepsilon_{y}$ are defined by the corresponding sub-phase-space determinants. Transverse coupling induces projection rms emittance increase while with the $4 \mathrm{D}$ emittance $\varepsilon_{4 d}$ is unchanged. It is more comprehensive to describe a coupled beam by introducing two eigenemittances $\varepsilon_{1}$ and $\varepsilon_{2}$ [18]. $\varepsilon_{4 d}$ keeps equal to the product of $\varepsilon_{1}$ and $\varepsilon_{2}$. For an uncoupled beam $\varepsilon_{x}=\varepsilon_{1}$, $\varepsilon_{y}=\varepsilon_{2}$ and $\varepsilon_{4 d}=\varepsilon_{1} * \varepsilon_{2}=\varepsilon_{x} * \varepsilon_{y}$. If the beam is coupled $\varepsilon_{4 d}=\varepsilon_{1} * \varepsilon_{2}<\varepsilon_{x} * \varepsilon_{y}[19]$. Based on the above simulation of extraction from FECR, calculated eigenemittances $\varepsilon_{1}=$ $0.59 \pi \mathrm{mm} \mathrm{mrad}$ and $\varepsilon_{2}=0.045 \pi \mathrm{mm} \mathrm{mrad}$. Consequently, the $4 \mathrm{D}$ emittance $\varepsilon_{4 d}=\varepsilon_{1} * \varepsilon_{2}=0.02655(\pi \mathrm{mm} \mathrm{mrad})^{2}$. However, the product of two projection rms emittances $\varepsilon_{x} * \varepsilon_{y}=0.1024(\pi \mathrm{mm} \mathrm{mrad})^{2}$, which is much larger than $\varepsilon_{4 d}$, indicating the beam is strongly coupled. 
As mentioned above, the second type of coupling resulting from the beam rotation can be dissociated if the beam rotates by a proper angle. Solenoid, which has normally been used as a prefocusing element after the ion source, provides a possibility to dissociate the transverse coupling by adding an extra rotation to the beam. However, one solenoid is insufficient to provide a proper focusing to the beam and meanwhile dissociate the coupling, while two solenoids will enhance the tuning flexibility. In the HIAF-LEBT design, two solenoids were arranged between the ion source and analyzing dipole. To verify the feasibility of beam decoupling, simulations were performed by using the particle distribution obtained from the extraction simulation. Figure 7 shows the simulated beam emittances at the end of the CSS by changing the field strengths and polarities of the two solenoids, in which the first solenoid Sol-1 was set to \pm 5000 Gs, and the second solenoid Sol-2 was scanned from -2200 to 2300 Gs. According to the plot, beam emittances with $B_{\text {Sol-1 }}=-5000$ Gs are much lower than those with $B_{\mathrm{Sol}-1}=5000$ Gs in both horizontal and vertical directions. In addition, the emittances change as a function of Sol-2 field. The emittances reach minimum while $B_{\text {Sol-2 }}=2200 \mathrm{Gs}$, meaning that under such conditions the transverse coupling of the beam is best relieved.

To further verify the emittance exchange effect, experiments have been done on the LECR4 ion source bench [20] at IMP. Similar with the FECR CSS, two solenoids are placed in the mixed beam section at LECR4. Experiments were carried out with the ion source optimized for production of $\mathrm{Xe}^{20+}$. By tuning the solenoid currents and polarities, the measured beam rms emittances are shown in Fig. 8. It should be noted that during the tests the beam current remained unchanged. Similar variation trends of the emittances with the simulations were obtained. The results indicate that the beam emittances depend upon the solenoid settings and a relatively smaller emittance could be achieved by carefully tuning the field

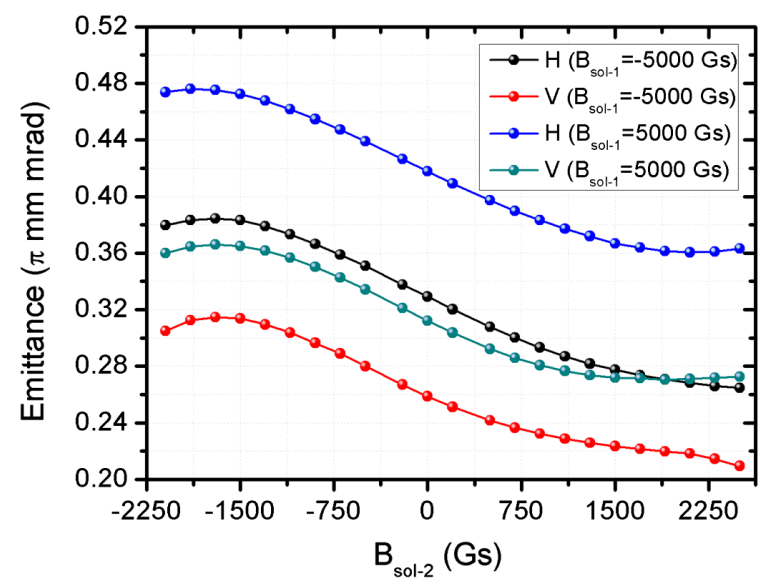

FIG. 7. Simulated beam emittances versus both field strengths and polarities of the two solenoids after the ion source.

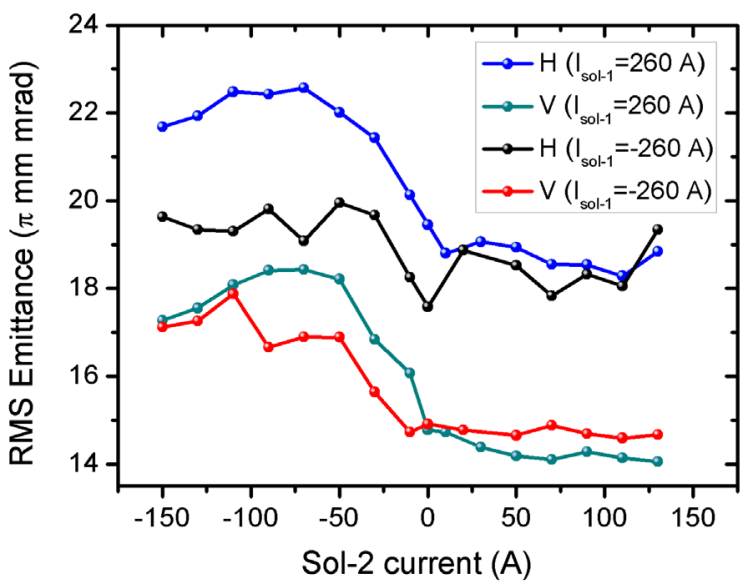

FIG. 8. Measured beam emittances at LECR4.

strengths and polarities of the two solenoids, meanwhile, beam focusing can also be satisfied.

Besides the emittance exchange function, studies [20] on the LECR4 platform have also indicated that two solenoids benefit beam matching for various species of ion beams so that the $Q / A$ resolution of the system can be improved.

\section{Space charge effect in the CSS}

At such a high beam intensity space charge effect has become a significant factor for beam dynamics design, especially in the CSS where multispecies ion beam current from the source may reach up to $20 \mathrm{emA}$. Fortunately, space charge compensation occurs when the beam particles interact with the residual gas molecules or bombard the beam pipe, creating slow secondary electrons. The electrons can be trapped in the beam potential and compensate the space charge effect. However, very few experimental measurements or numerical simulations give an evaluation of the space charge compensation degree for low energy highly charged heavy ion beams. Usually, a conventional trick is to perform the simulations with different space charge compensation factors to guarantee a robust design.

In the simulation for the CSS of FECR the initial mixed beam was simplified to include 20 different ion species containing uranium and oxygen (supporting gas) ions, as shown in Fig. 9. The beam current was scaled up according to the charge state distribution from VENUS (in Ref. [12]) so that the $U^{35+}$ beam current reached up to $2 \mathrm{emA}$, producing a total current of $20 \mathrm{emA}$. All the ion beams are assumed to have the same initial conditions and start with a fourdimensional water bag model. The Twiss parameters were obtained from the beam extraction simulation with IBSimu, as described in the previous section, with normalized rms emittance of $0.24 \pi \mathrm{mm}$ mrad. Beam transmission simulations were carried out through the CSS for different space charge compensation degrees, $70 \%, 50 \%$ and $30 \%$ with TRACK code [21]. Figure 10 shows the simulated particle 


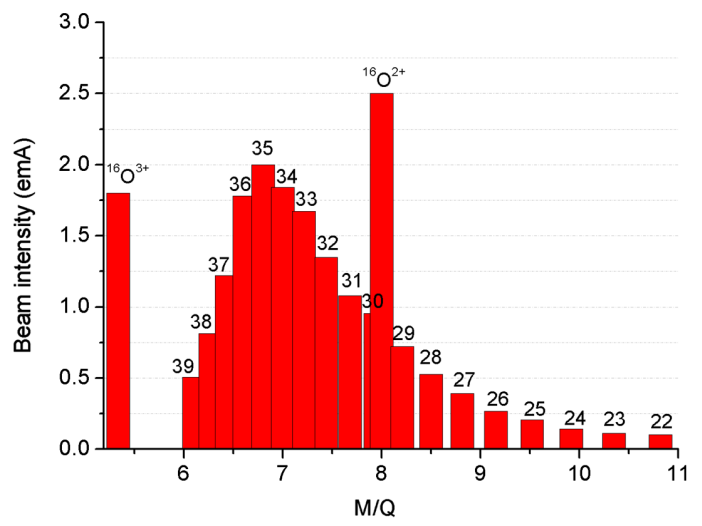

FIG. 9. Charge state distribution of the initial mixed beam $\left({ }^{238} \mathrm{U}\right.$ and $\left.{ }^{16} \mathrm{O}\right)$ in the simulation.

distribution of different charge states at the end of the CSS line where the charge selecting slit is located under different compensation degrees. It is obviously seen that there is a good separation between ions with different mass-to-charge ratios when the mean compensation factor is $70 \%$. While with $50 \%$ compensation the objective ion $\mathrm{U}^{35+}$ can be barely separated out. However, serious overlap between adjacent ion species and much more stray particles appear in the plane when the compensation is as low as $30 \%$. These simulations indicate that space charge effect has a vital impact on the beam transmission and beam line design by increasing the beam spots in the focal plane and reducing the momentum resolution of the CSS system. However, the question is: how much is the compensation factor?

To investigate the space charge effect and compensation in the ECR source associated CSS line, a series of experiments were implemented based on the measurements of beam emittance at SECRAL and SECRAL-II ion source benches. There are three groups of experimental data. The first one is for optimizing ${ }^{40} \mathrm{Ar}^{9+}$ beam production with SECRAL-II. We measured the beam emittances after the CSS both in the horizontal and the vertical planes for beam intensity of ${ }^{40} \mathrm{Ar}^{9+}$ from 0.32 to $1.75 \mathrm{emA}$, with total beam current (drain current from source) $I_{0}$ increasing from 4 to $14 \mathrm{emA}$. Figure 11(a) illustrates the dependence of the normalized rms emittances on the beam intensities. The second experiment was implemented by optimizing ${ }^{86} \mathrm{Kr}^{18+}$ beam in the same way, and the third one was for optimizing ${ }^{209} \mathrm{Bi}^{31+}$ beam at the SECRAL source. The results are shown in Figs. 11(b) and 11(c), respectively. In these experiments the background pressures in the beam lines were in a range of between $1 \times 10^{-7}$ and $3 \times 10^{-7}$ mbar. All these measurements indicate that even up to a few hundreds of $e \mu \mathrm{A}$ or higher current, there is no significant increase of the beam emittance with the beam intensity, and even presenting a phenomenon that beam emittance may decrease while beam intensity increases. In other words, beam emittance is slightly dependent on the beam intensity, but largely depends upon the source tuning and the ECR plasma conditions.

To further investigate the space charge compensation degree, simulation towards SECRAL CSS was performed. Figure 12 shows a measured CSD with the ion source optimized for production of $\mathrm{Bi}^{31+}$. Similarly to the above simulation, 20 different ion species were used as the initial mixed beam, as shown in Fig. 13. The beam current of each ion was scaled up according to Fig. 12 so that the total current is equal to the source drain current of $13 \mathrm{emA}$. Simulated particle distributions at the end of CSS, under space charge compensation factors of $70 \%$, $50 \%$ and 30\%, are shown in Fig. 14. It is clearly observed that the objective ion $\mathrm{Bi}^{31+}$ can just be separated from the adjacent charge state under $70 \%$ compensation, while a lower degree of the compensation will lead to an overlap with adjacent ion species. However, according to Fig. 12, a very good resolution between different charge states was obtained because the beam current could reach zero between neighboring current peaks. This comparison between experiment and simulation implies that the overall space charge compensation degree would be no less than $70 \%$.

In conclusion, space charge effect is not a primary reason of beam quality degradation as expected because of good
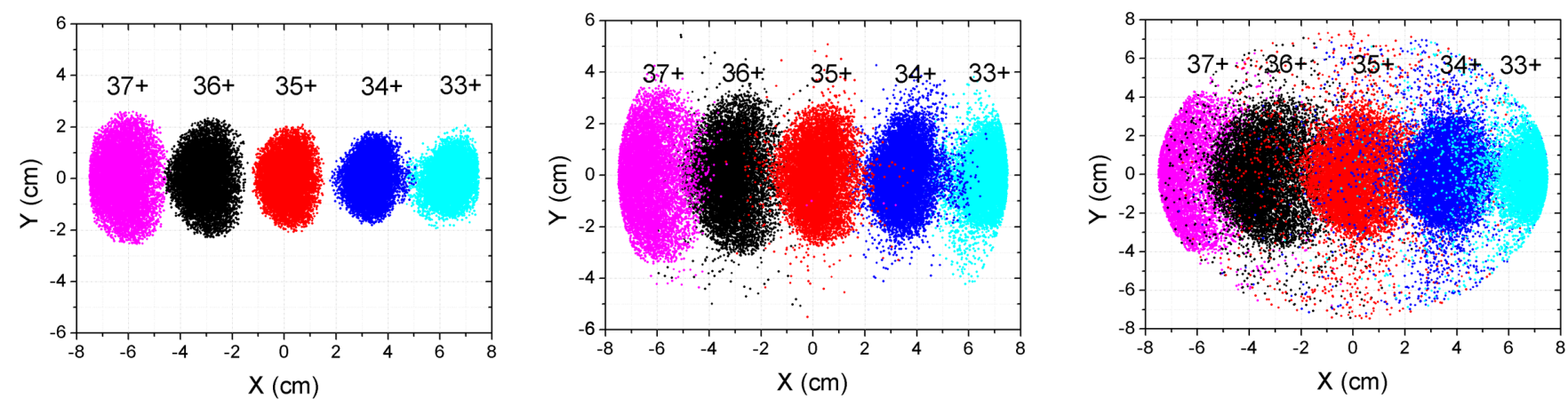

FIG. 10. Simulated particle distributions of different charge states at the end of CSS under space charge compensation degrees of $70 \%$ (left), 50\% (middle) and 30\% (right). 

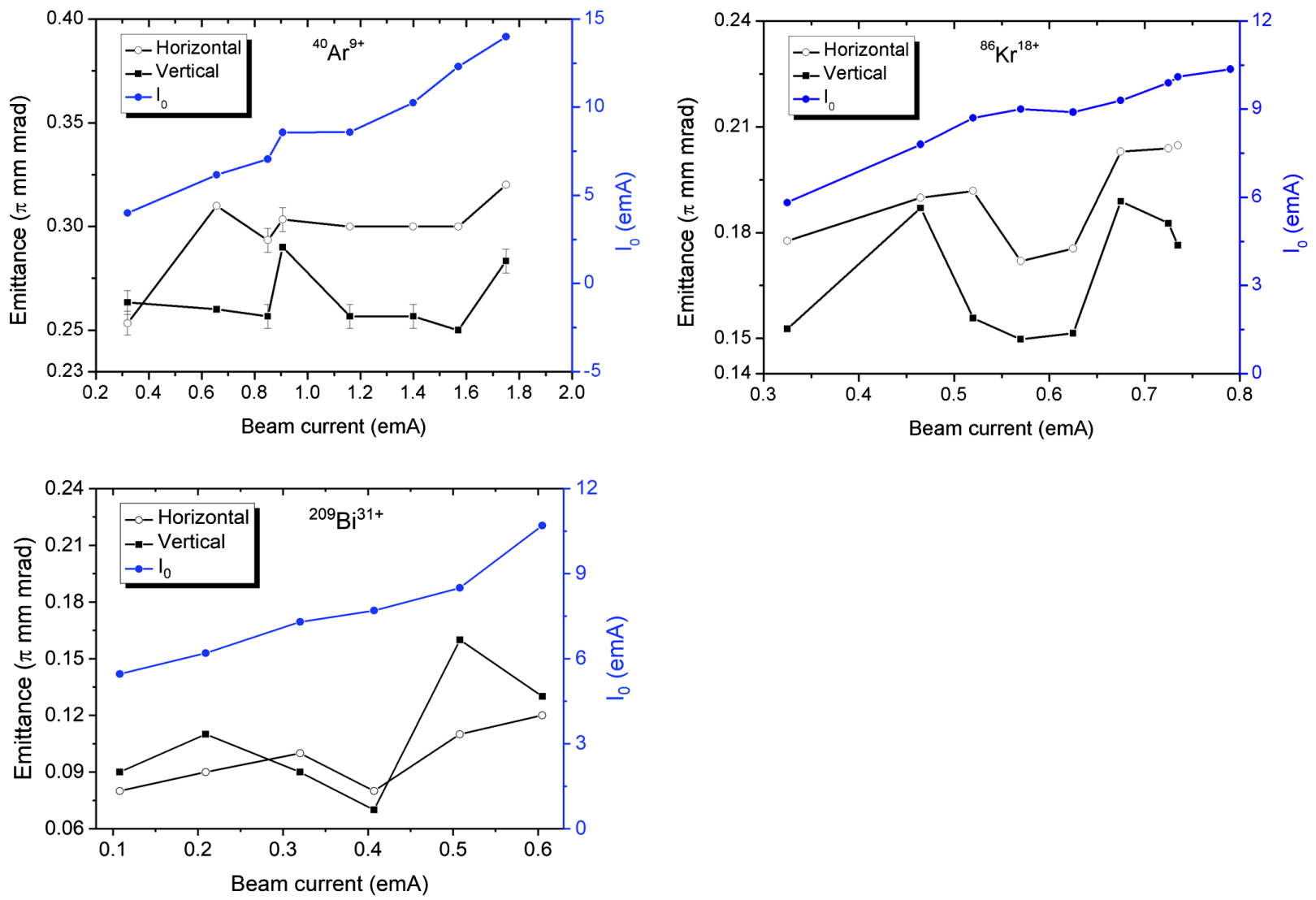

FIG. 11. Measured beam emittances versus beam intensities ( $I_{0}$ is the total beam current from the source).

compensation in the ECR CSS lines. Beam quality is mainly determined by the ion source tuning and plasma conditions. In the CSS design of highly charged heavy ion beams it is safe to set the overall space charge compensation factor to $70 \%$.

The level of space charge compensation was directly measured by using a so-called retarding field analyzer after the analyzing magnet of SuSI ECR ion source at Michigan

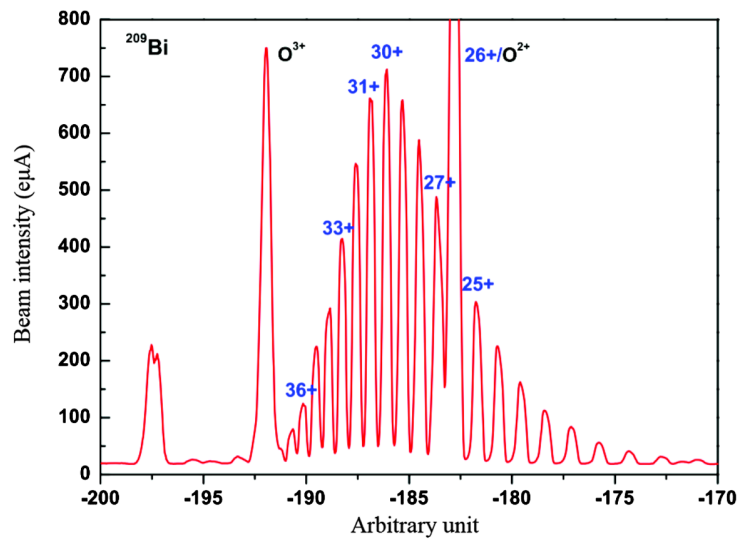

FIG. 12. CSD for bismuth and oxygen (supporting gas) ions.
State University. The measurements suggest overall low compensation factors $(0 \%-60 \%)$ at this location [22]. However, in the CSS the measurement is hard to do due to the multispecies in the beam. An extended theoretical mode [23] was introduced in their study. Based on the theoretical mode they predicted that in the CSS the compensation could reach a high level, which agrees with our prediction in this paper.

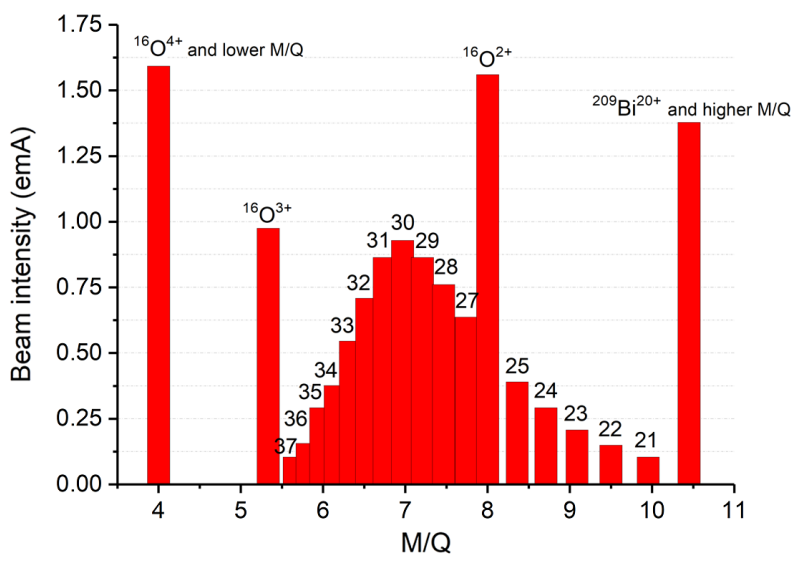

FIG. 13. Charge state distribution of the initial mixed beam ( $\mathrm{Bi}$ and $\mathrm{O}$ ) in the simulation. 

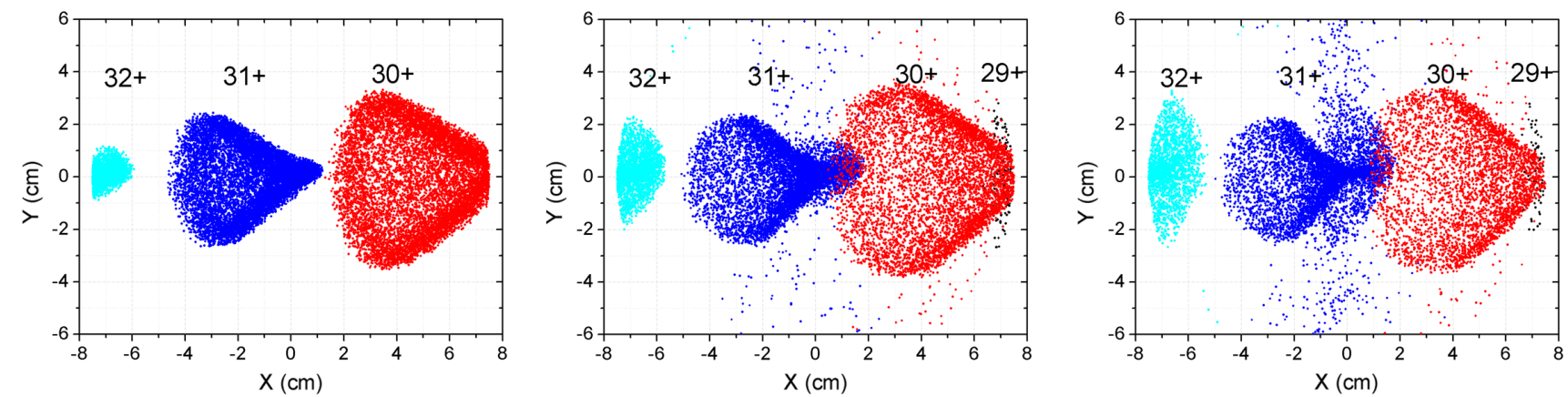

FIG. 14. Simulated particle distributions at the end of CSS under space charge compensation degrees of 70\% (left), $50 \%$ (middle) and $30 \%$ (right).

\section{LOW ENERGY BEAM TRANSPORT}

\section{A. Achromatic design}

Design of the LEBT line is based on an achromatic system composed of two quadrupole triplets surrounded by two $90^{\circ}$ dipoles. Beam optics through the LEBT is firstly implemented by using TraceWin code [24] with matrix mode. The $2 *$ rms envelope and dispersion function along the LEBT from the source exit to the RFQ entrance are shown in Fig. 15, which shows an achromatic condition is achieved based on a reasonable envelope distribution along the LEBT line.

\section{B. Multiparticle tracking simulation}

Further multiparticle tracking was carried out with TRACK code by using the initial particle distribution (shown in Fig. 4) obtained from the extraction simulation. In this simulation, 3D field maps were used for modeling the magnetic elements of the beam line, except for the dipoles which were modeled with a hard edge model. Simulated phase space distributions after the CSS are shown in Fig. 16. The simulations have a very similar beam profile with the measurements at VENUS for production of $311 \mathrm{e} \mu \mathrm{A}$ $\mathrm{U}^{34+}$ beam with total extraction current of $7.5 \mathrm{emA}$ under $28 \mathrm{GHz}$ microwave heating [25], as shown in Fig. 17. The irregular shape of the distortions in the phase spaces could be mainly due to the initial beam configuration from the source, and also aberrations from the solenoid lenses and the analyzing magnet. Thanks to the emittance exchange effect of the two solenoids after the source the emittances were reduced from $0.32 \pi \mathrm{mm}$ mrad to $0.27 \pi \mathrm{mm} \mathrm{mrad}$ in horizontal and to $0.21 \pi \mathrm{mm}$ mrad in the vertical. Even so, the simulated emittances are much larger than those in the measurement at VENUS where the horizontal emittance is of
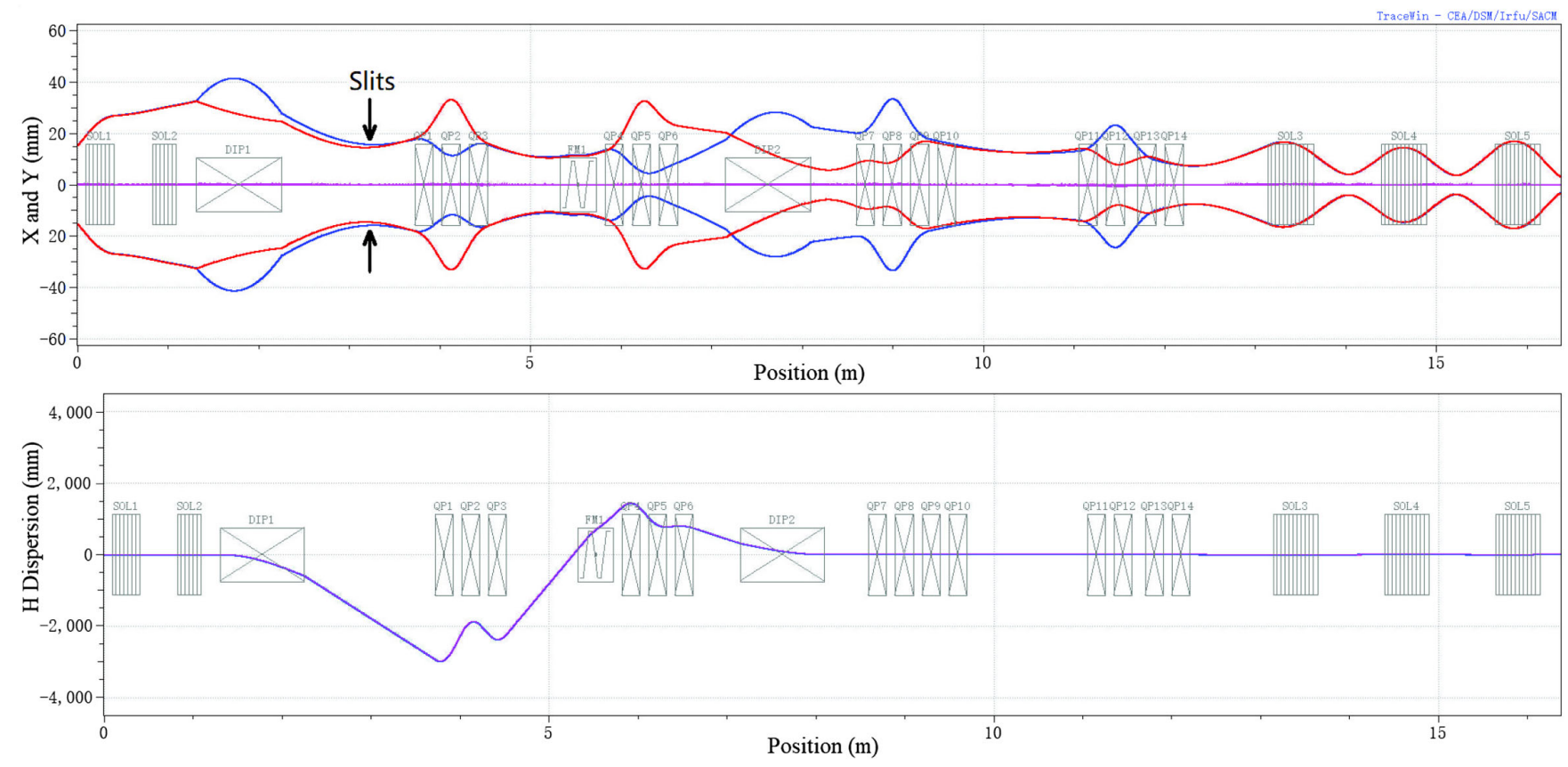

FIG. 15. Simulated beam $2 *$ rms envelope and dispersion function along the LEBT by TraceWin. 

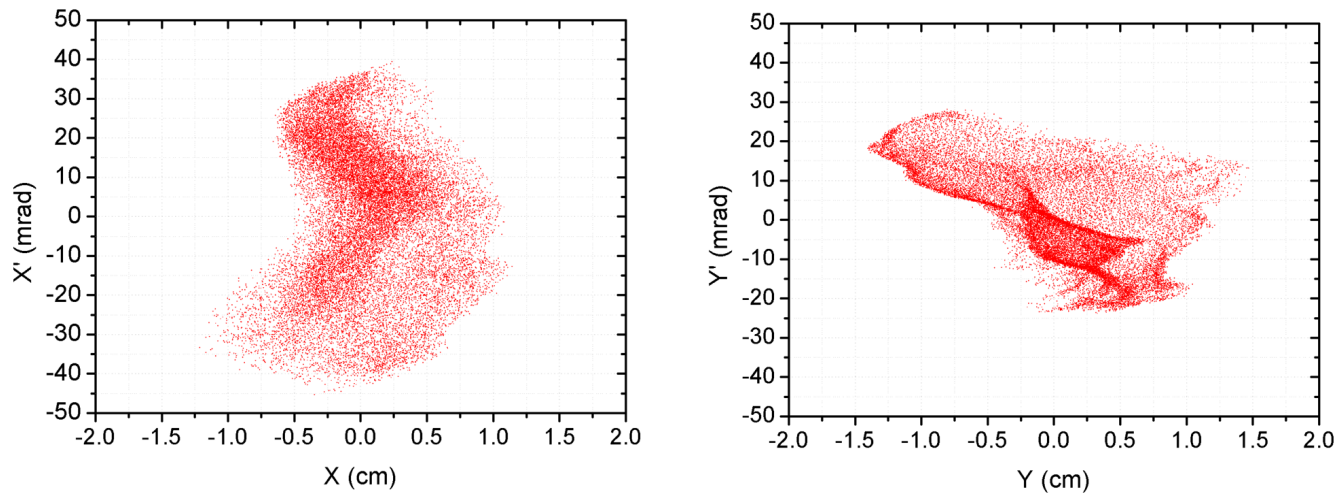

FIG. 16. Simulated phase-space distributions after the CSS using the initial particle distribution obtained from the extraction simulation.
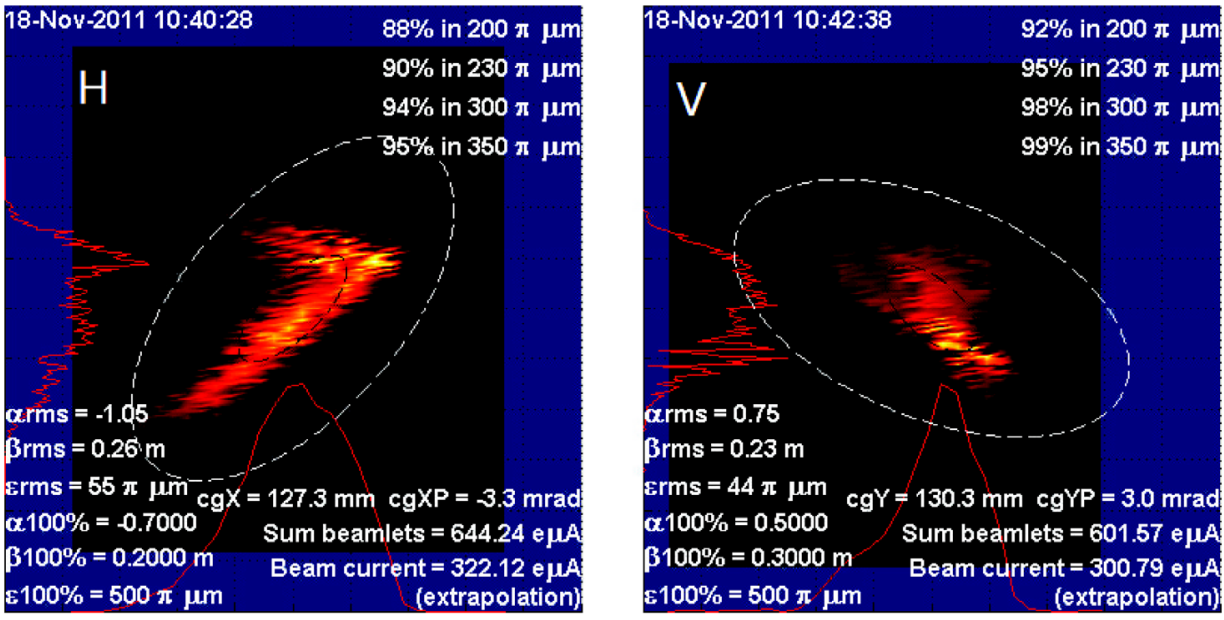

FIG. 17. Measured beam emittance of $\mathrm{U}^{34+}$ with VENUS (in Ref. [25]).

about $0.14 \pi \mathrm{mm} \mathrm{mrad}$ and vertical of $0.11 \pi \mathrm{mm} \mathrm{mrad}$. It is reasonable because the magnetic field and beam intensity are much stronger in FECR than those in VENUS.

Starting with the realistic particle distribution, beam transmission through the LEBT line was implemented for the ion current of $2 \mathrm{emA}$ with $70 \%$ space charge compensation, except in the acceleration tube and MHB where space charge compensation is destroyed by the electrical field. The beam envelope is illustrated in Fig. 18. The ion beam is delivered by the LEBT with a moderate size of envelope and successfully matched to the RFQ. However, particle distributions in the phase spaces at the RFQ entrance, as shown in Fig. 19, present a badly distorted beam with outstretched particle "tails" beyond the boundary of the RFQ acceptance (shown with the red ellipse). The beam tails would be lost in the RFQ or even in the downstream superconducting section. Therefore, development of a collimation system in the LEBT is necessary.

As shown in Fig. 2, a collimation channel is designed based on three apertures successively arranged between two solenoids (PSN). The front PSN is used to drive the beam to rotate in the phase space. A phase advance of approximately 45 degrees between each aperture is needed so that a total phase advance of 90 degrees can be produced

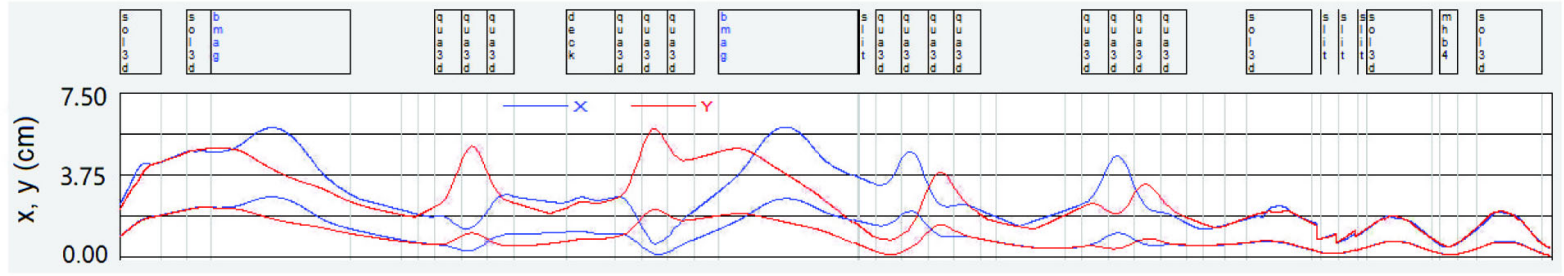

FIG. 18. TRACK simulation of the beam envelope with the realistic particle distribution. 

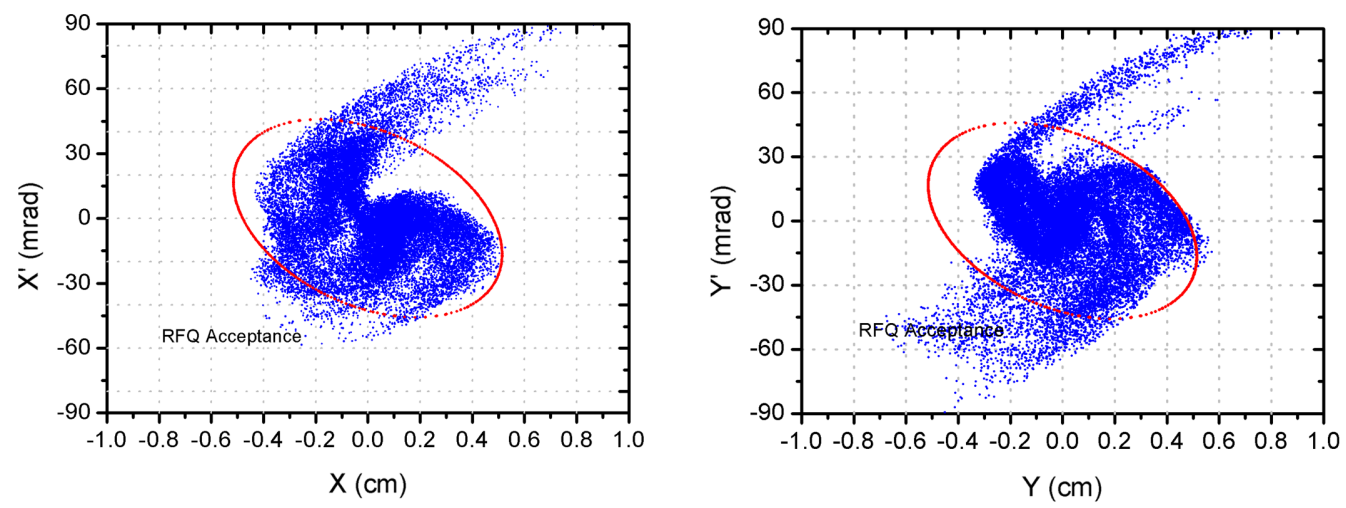

FIG. 19. Particle distributions in the phase spaces at the RFQ entrance without collimation.

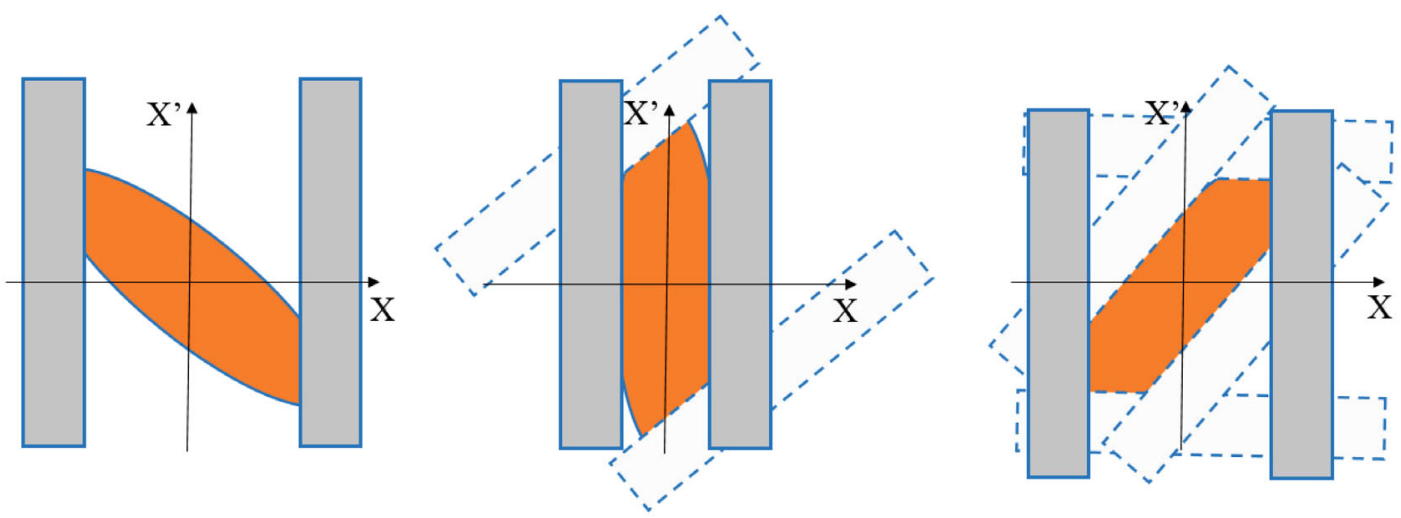

FIG. 20. Principle of beam collimation.

from the first aperture to the third aperture. Figure 20 shows the principle of phase space rotation and cutting of the particle distribution in the two-dimensional subphase space. To create an expected phase advance at each aperture, the upstream quadrupoles should be tuned to create a certain orientation of beam phase space ellipse before the PSN.

After experiencing three successive cuts, approximately $20 \%$ of the particles are eliminated (1.6 emA is left). Particle distribution in the phase spaces at RFQ entrance are

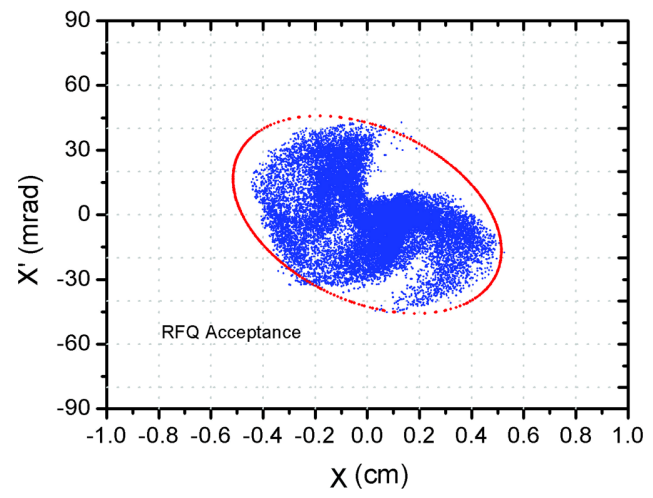

shown in Fig. 21. Although the beam is still distorted, most of the beam tails are stopped by the collimators and a few particles go beyond the RFQ acceptance. Introduction of the collimation channel benefits the beam emittance reduction from 0.27 to $0.16 \pi \mathrm{mm}$ mrad in the horizontal and from 0.31 to $0.15 \pi \mathrm{mm} \mathrm{mrad}$ in the vertical, respectively, indicating that $20 \%$ of the beam tails contribute to more than $70 \%$ of the emittance.

Beam tuning of the LEBT line should be based on the beam quality measurement after charge selection where

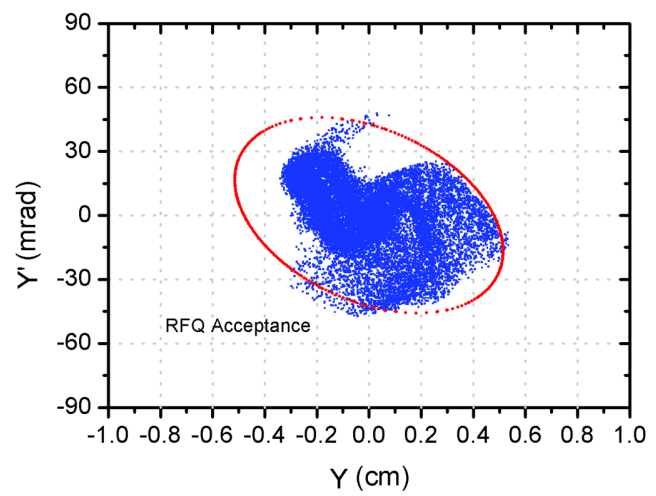

FIG. 21. Simulated particle distributions at the RFQ entrance with collimator on. 
TABLE I. Basic design specifications of LEAF-RFQ.

\begin{tabular}{lc}
\hline \hline Duty cycle & $100 \%$ \\
Operating frequency $(\mathrm{MHz})$ & 81.25 \\
Resonant cavity & 4 -vane \\
Input particle energy $(\mathrm{keV} / \mathrm{u})$ & 14 \\
Output particle energy $(\mathrm{keV} / \mathrm{u})$ & 500 \\
Maximum vane voltage $(\mathrm{kV})$ & $70\left(\mathrm{U}^{34+}\right)$ \\
cw rf power $(\mathrm{kW})$ & $60\left(\mathrm{U}^{34+}\right)$ \\
Peak field at electrode surface & 1.57 Kilpatrick units \\
Length of the RFQ vane $(\mathrm{cm})$ & $\sim 596.4$ \\
\hline \hline
\end{tabular}

two Allison scanners are available. Starting with the measured particle distribution, the subsequent simulation with multiparticle tracking could well predict the beam phase space profile at any location in the LEBT [26]. By setting the magnets on the basis of simulation, beam quality measurement before the collimation channel helps finetuning of the magnets to create an expected orientation of beam phase space ellipse so that the collimator plays its due role. Here we use a pepper-pot device for fast emittance measurement. Adjustment of the collimator apertures is to achieve a high RFQ transmission efficiency with as low as possible beam loss. After collimation cutting and before entering the RFQ, the beam quality will be measured again just before the last focusing PSN before the RFQ.

\section{THE RFQ AND BEAM COMMISSIONING}

\section{A. LEAF-RFQ and beam commissioning}

LEAF-RFQ [27-28], which has been constructed at IMP and successfully commissioned with several beams, such as $\mathrm{He}^{2+}(Q / A=1 / 2), \mathrm{C}^{4+}(Q / A=1 / 3), \mathrm{He}^{+}(Q / A=1 / 4)$, $\mathrm{Kr}^{13+}(Q / A=1 / 6.6), \mathrm{N}^{2+}(Q / A=1 / 7)$, etc., is a prototype of HIAF-RFQ. Table I lists the basic design specifications of LEAF-RFQ. Figure 22 shows the main RFQ parameters as a function of cell number. The focusing strength was held constant $(B=4.34)$ along the structure leading to a constant average aperture radius of $5.8 \mathrm{~mm}$, keeping the capacitance independence of longitudinal position to facilitate cavity tuning. The transverse geometry of the vane tip is circular with the same transverse radius of curvature throughout the RFQ making fabrication easier. The ratio between the vane-tip radius and the average radial aperture was chosen to be 0.75 as a compromise between the peak voltage and the effect of multipoles.

To obtain the lowest possible output emittance and shorter vane length, an external MHB was employed upstream of the RFQ, and the RFQ only accepts the well bunched core particles for further acceleration avoiding capture of the small fraction particles in the tails of the distribution. Since the beam is already bunched at the entrance of the RFQ, an initial synchronous phase of $-45^{\circ}$ and a modulation factor of 1.05 were chosen so that the longitudinal acceptance is truncated. Following the development in FRIB at MSU [29], a single gap MHB with three harmonics was adopted. The three resonant frequencies are produced via two quarter-wave resonators: one resonator is driven at its fundamental mode at $40.625 \mathrm{MHz}$ (half of RFQ frequency) and its first higher-order mode (121.875 MHz), while the other is driven only at its fundamental mode of $81.25 \mathrm{MHz}$. The side cross section of LEAF-MHB is shown in Fig. 23. The MHB aperture diameter of smaller opening is $22 \mathrm{~mm}$ and the gap between the electrodes is $8 \mathrm{~mm}$. With two higher harmonics linearizing the voltage ramp the $\mathrm{MHB}$ is expected to produce an approximately linear saw-tooth modulation of the beam energy. The expected longitudinal distribution and the initial reduced-size separatrix are shown in Fig. 24 [30-31].

LEAF-RFQ was assembled in December 2017 and the cavity rf conditioning finished in February 2018. The conditioning in $\mathrm{cw}$ mode up to $75 \mathrm{~kW}$ (1.1 times the required maximum rf power) went smoothly, consuming only

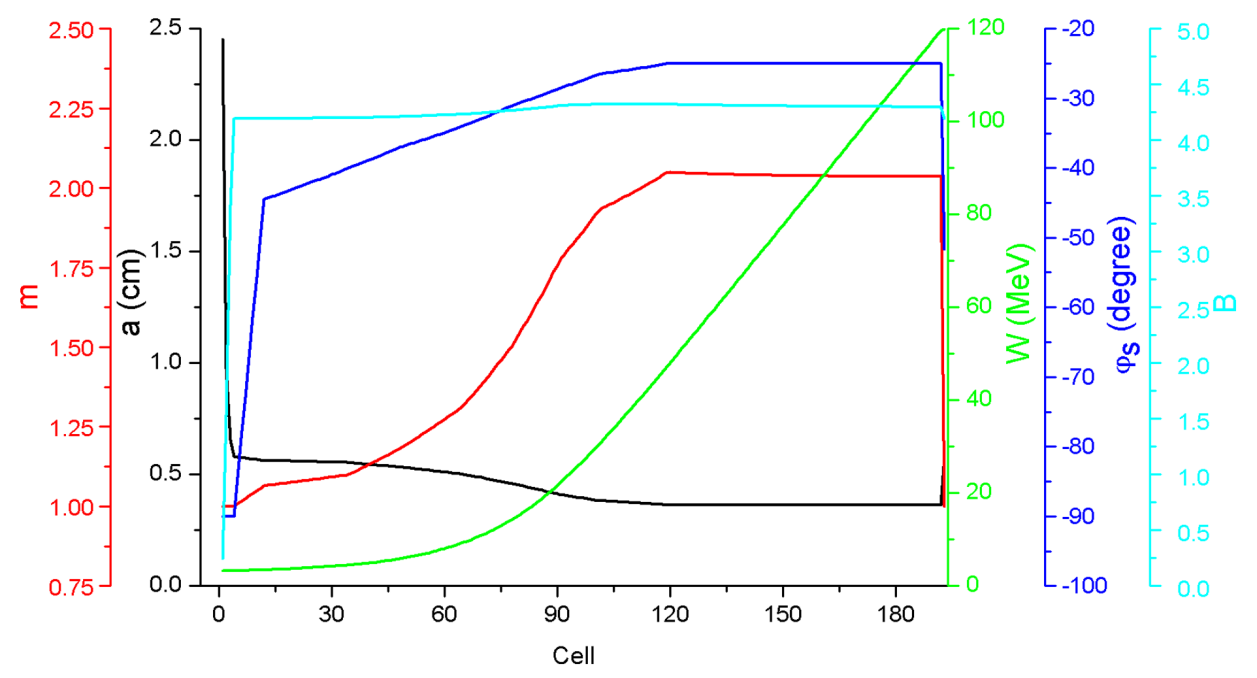

FIG. 22. Main parameters as a function of cell number for LEAF-RFQ. 


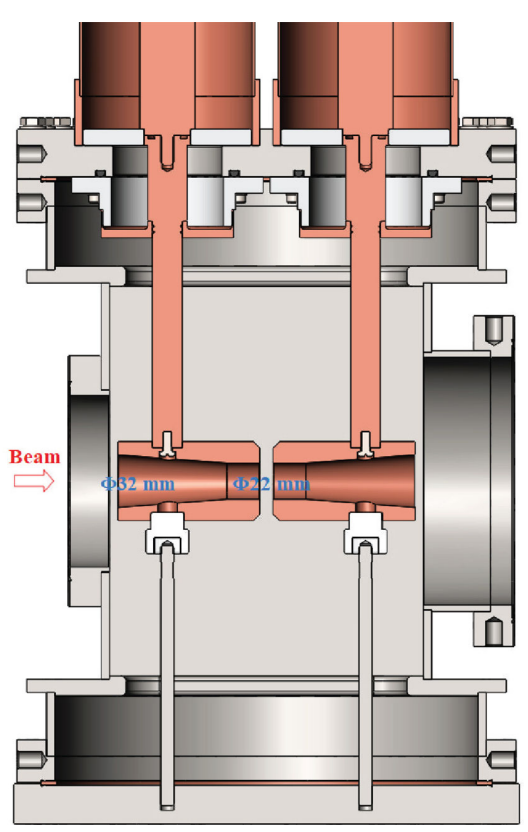

FIG. 23. Side cross section of the LEAF-MHB.

44 hours. The whole complex installation, excepting the MHB, was finished in May 2018, and the beam commissioning started soon after that. Early beam commissioning and characteristic measurement without MHB were reported in [26,32]. The MHB was installed in September 2018. The beam transmission increased to the designed value with MHB operational. The $\mathrm{N}^{2+}$ beam was used as a substitute of $\mathrm{U}^{34+}$ to evaluate the accelerator performance due to the same rigidities. Figure 25 shows the measured and simulated acceleration efficiencies of the RFQ for $\sim 100 \mathrm{e} \mu \mathrm{A} \mathrm{N} \mathrm{N}^{2+}$ beam under different MHB operation conditions. The full transmission efficiency, including nonaccelerated current, was measured by two ac current transformers (ACCT)

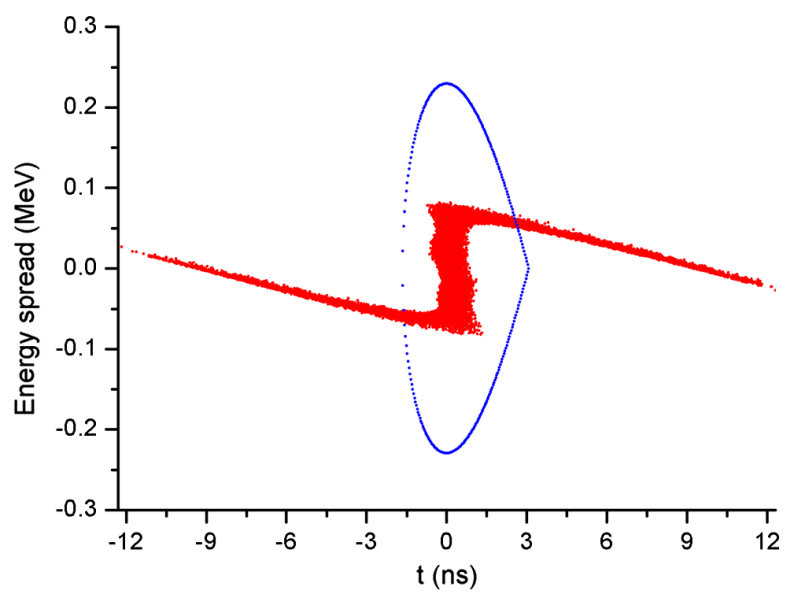

FIG. 24. Expected longitudinal distribution at the RFQ entrance of the beam bunched by the MHB and the initial reduced-size separatrix of the longitudinal motion.

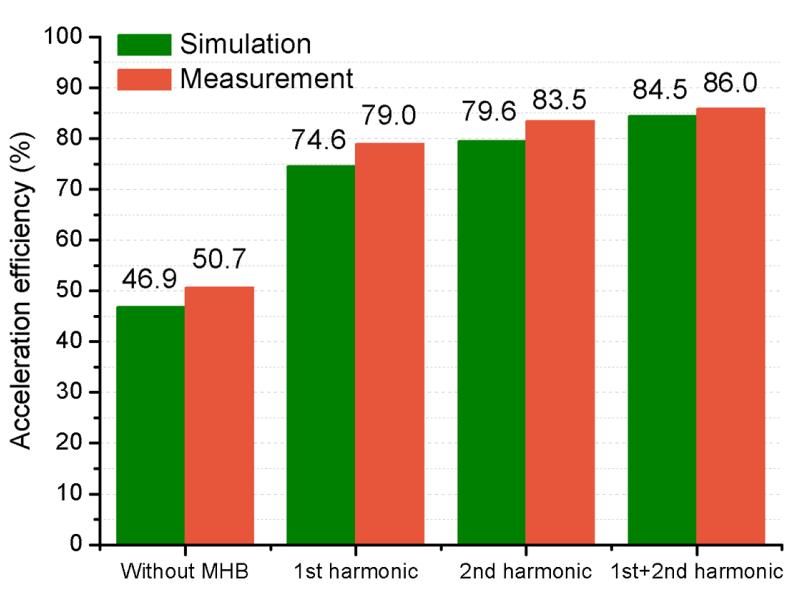

FIG. 25. Measured and simulated acceleration efficiencies of the RFQ for $\sim 100 \mathrm{e} \mu \mathrm{A} \mathrm{N}^{2+}$ beam under different MHB operation conditions.

situated on both sides of the RFQ, as illustrated in Fig. 26. The measured transmission was higher than $97 \%$ which is similar to the simulated value. The acceleration efficiency is the ratio of the beam currents at Faraday cup (FC4) located after the MEBT quadrupole triplet and ACCT-1 before the RFQ. Simulations predict that the nonaccelerated particles would be overfocused and lost in the triplet focusing channel due to the widely different rigidity from the synchronous particles. Good agreement between the measurements and simulations was demonstrated, while the small difference can be due to the measurement errors and the deviations of the simulation model. However, due to the power limitation of the amplifier, the third harmonic with frequency of $121.875 \mathrm{MHz}$ hardly has contributions to the beam intensity in the measurement. To investigate the validity of the third harmonic, the measurement was conducted with the designed minimum $M / Q$ ion $\mathrm{He}^{2+}$ which requires a much lower field in the center gap, decreasing the required rf power. As shown in Fig. 27, with the third harmonic working the acceleration efficiency increased by $2 \%$, comparing with that using two harmonics. Since the design ion for the RFQ has $M / Q=7$, the acceleration efficiency for $\mathrm{He}^{2+}$ is generally lower than $\mathrm{N}^{2+}$.

To better understand the bunching effect of the MHB to the beam, Fig. 28 shows the particle distribution in longitudinal phase space after being bunched by the first harmonic (40.625 MHz), the second harmonic $(81.25 \mathrm{MHz}), 1 \mathrm{st}+2 \mathrm{nd}$ harmonics and $1 \mathrm{st}+2 \mathrm{nd}+3 \mathrm{rd}$ harmonics, respectively. The orientation of the beam is to match the orientation of the RFQ acceptance to maximize the acceleration efficiency. Figure 29 shows the beam longitudinal bunch shape detected by a fast Faraday cup (FFC, as shown in Fig. 26, after a drift subsequent to the RFQ) which has a time resolution of 80 ps (bandwidth limitation of $12.5 \mathrm{GHz}$ ). It is observed that primary bunches 


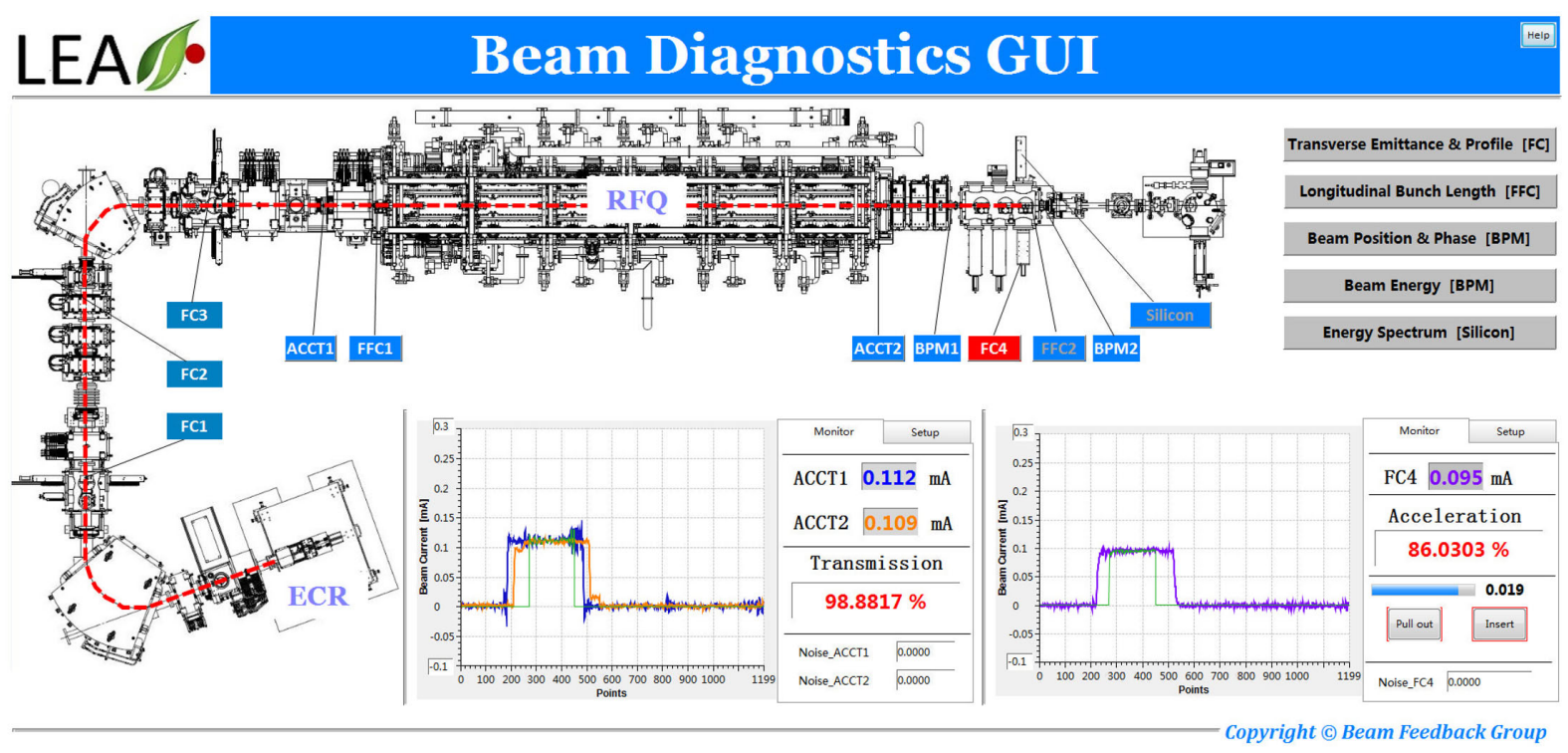

FIG. 26. Interface of the beam diagnostics.

and satellite bunches are arranged alternatively in time. That is because the fundamental frequency of MHB is half of the frequency of RFQ. The measurement also indicated a hollow bunch profile. The simulations, shown in Fig. 30, give the explanation: at low beam current the space charge force is not enough to make the S-shape particle distribution in the longitudinal phase-space dispersive [33]. The measured full width at half maximum (FWHM) of the bunch length was about $1.1 \mathrm{~ns}$, which was slightly larger than the simulation (see Fig. 30, FWHM $\sim 0.8 \mathrm{~ns}$ ).

\section{B. A proposal of new prebunching scheme}

The performance of LEAF-RFQ has demonstrated successful system design. A similar structure would be adopted by HIAF. However, a new prebunching scheme

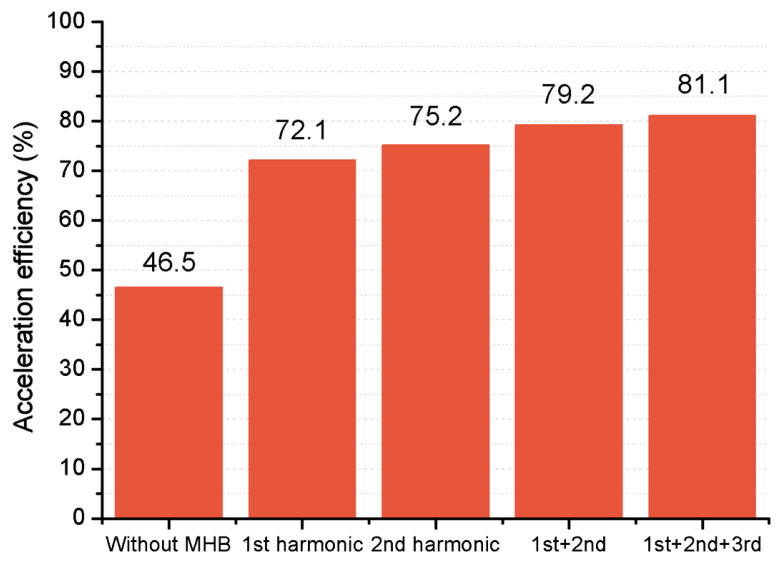

FIG. 27. Measured acceleration efficiencies of the RFQ for $\mathrm{He}^{2+}$ beam under different MHB operation conditions. that has a fundamental frequency equal to the RFQ frequency has been considered in HIAF to avoid satellite bunches as shown in Fig. 29. The energy gain of a charged particle passing through a buncher depends on the transit time factor (TTF) which is a function of both the particle velocity and harmonic number. The difficulty to increase the frequency of the MHB lies in the lower TTF for higher frequency harmonic. The TTF for a single accelerating gap can be given by

$$
T=\frac{\int E(0, z) \cos (2 \pi z / \beta \lambda) d z}{\int E(0, z) d z},
$$

where $E(0, z)$ is the electric field along the axis, $\beta$ is the particle velocity and $\lambda$ the wave length. To improve the TTF, considering a MHB with the electrodes separated by $4 \mathrm{~mm}$ with center aperture diameters of $20 \mathrm{~mm}$, the axial field distribution obtained from electromagnetic studio (EMS) [34] simulation is shown in Fig. 31. Figure 32 presents the dependence of TTF on the frequency. Higher frequency results in lower TTF, indicating higher $\mathrm{rf}$ power consumption. That is why we chose half of the RFQ frequency as the fundamental frequency of MHB in LEAF.

Although the MHB resonator operating with three frequencies could produce an approximately linear sawtooth in the voltage as a function of time [35], the beam energy may not gain a sawtooth modulation as shown in Fig. 24 where the longitudinal space charge effect of the beam is neglected. By taking longitudinal space charge effect into account, after being bunched by the MHB (LEAF-MHB) the simulated particle distribution in the longitudinal phase space at RFQ entrance for $1.6 \mathrm{emA}$ 

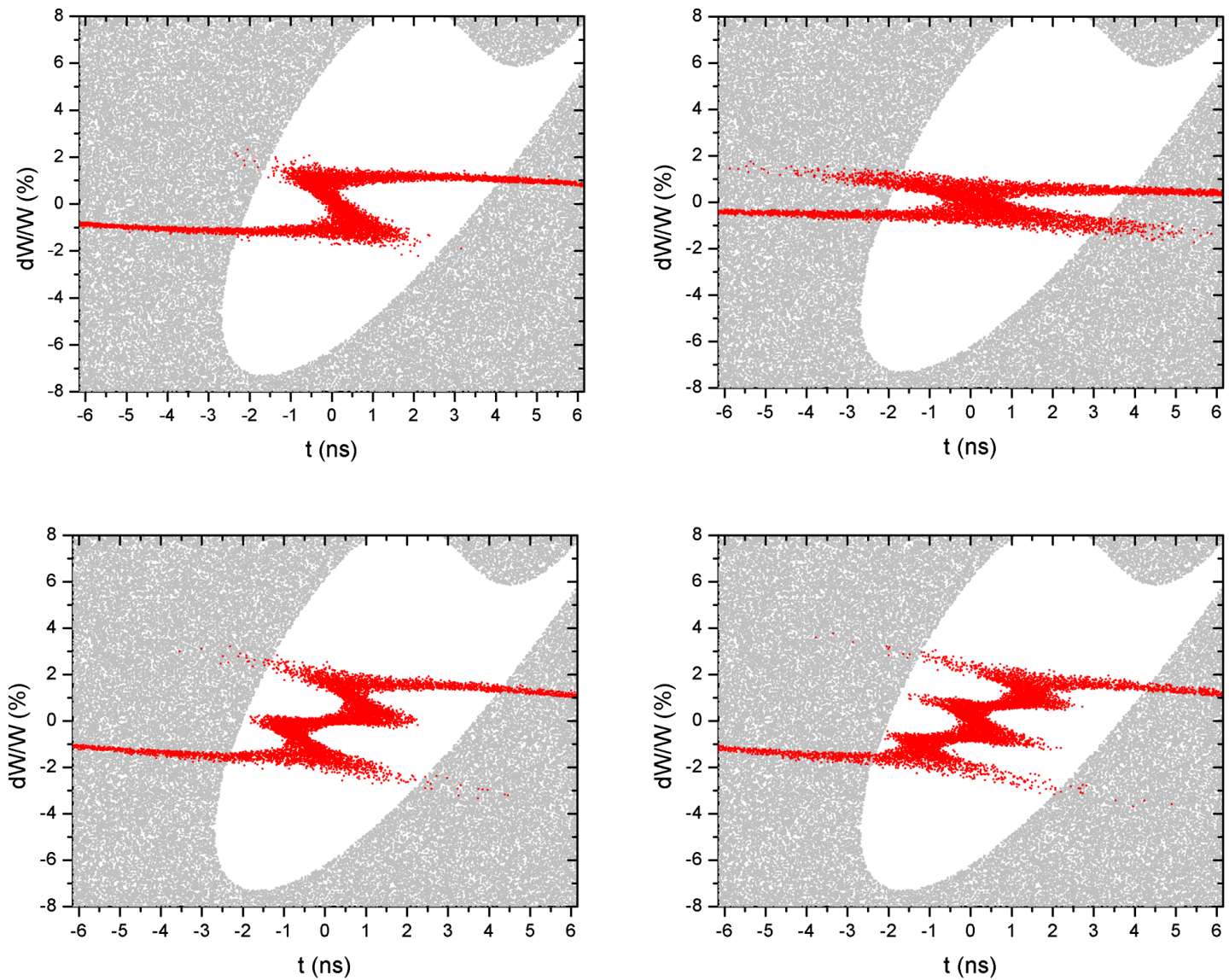

FIG. 28. Simulated particle distributions in longitudinal phase space after being bunched by the first harmonic, the second harmonic, $1 \mathrm{st}+2 \mathrm{nd}$ harmonics and $1 \mathrm{st}+2 \mathrm{nd}+3 \mathrm{rd}$ harmonics, respectively.

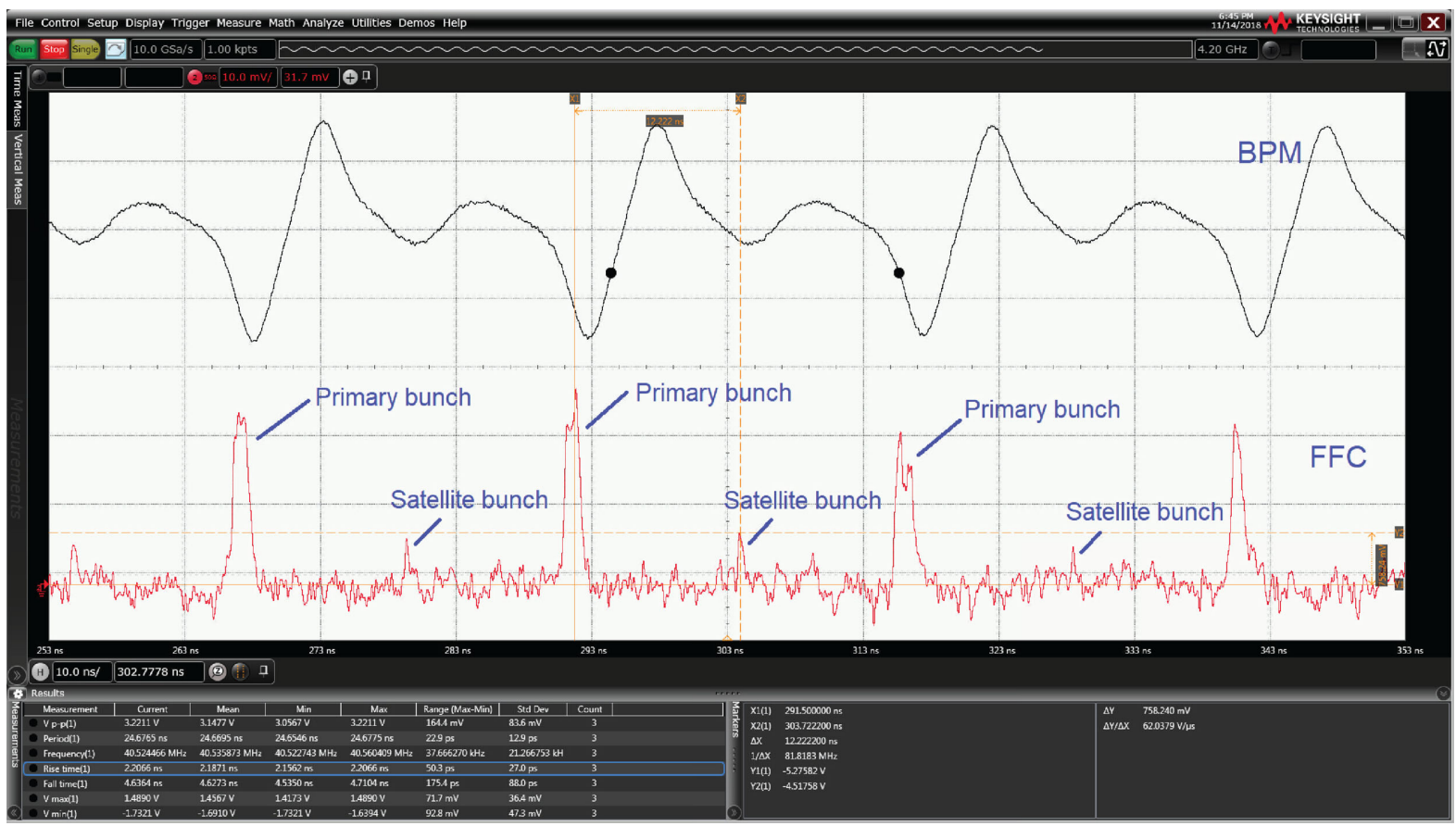

FIG. 29. Measured beam longitudinal bunch shape by a fast Faraday cup. 

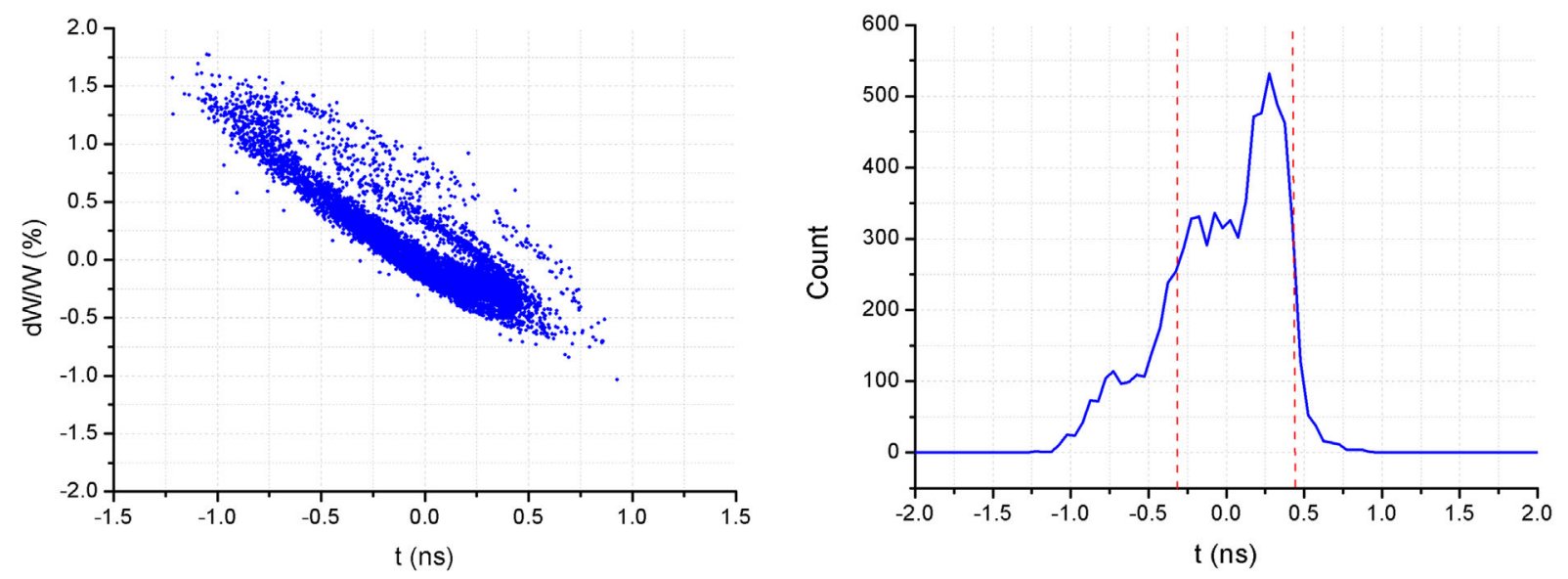

FIG. 30. Simulated beam longitudinal distribution and bunch shape.

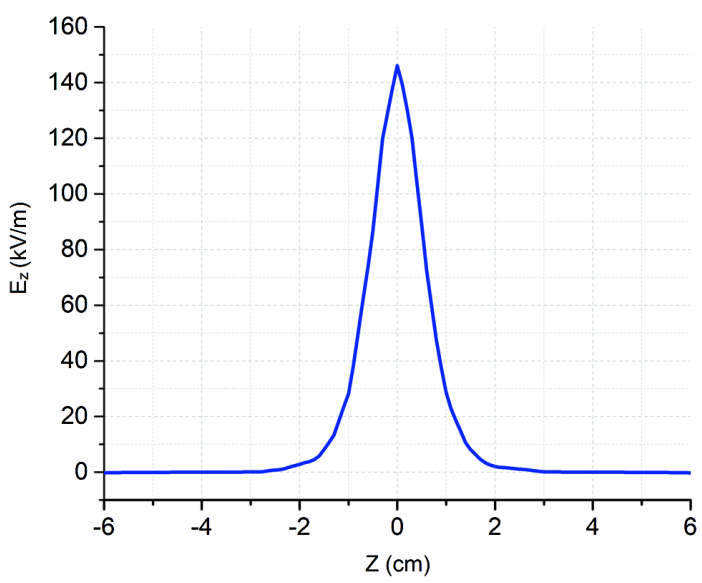

FIG. 31. MHB longitudinal electric field along the beam axis.

$\mathrm{U}^{35+}$ beam with energy of $14 \mathrm{keV} / \mathrm{u}$ is shown in Fig. 33 . Unlike the simulation in Fig. 24, the beam in longitudinal phase space presents a serious distortion with nonlinear effect [36].

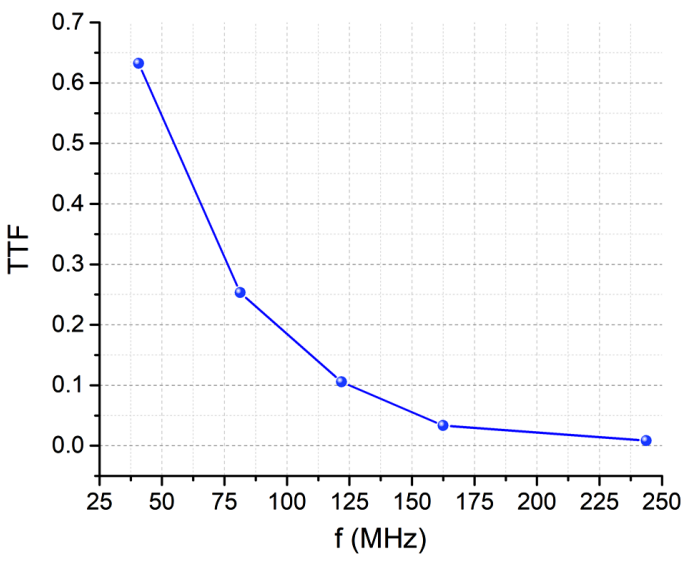

FIG. 32. Frequency versus TTF.
Now that a sawtooth-like waveform of energy spread cannot be obtained for intense beam due to the impact of the space charge effect, a new prebuncher scheme containing two frequencies of 81.25 and $162.5 \mathrm{MHz}$ is proposed in HIAF. By matching the orientation of the RFQ acceptance Fig. 34 shows a series of bunched beams by a three-harmonic-buncher $(40.625+81.25+$ $121.875 \mathrm{MHz})$ and a two-harmonic buncher $(81.25+$ 162.5 MHz) respectively. The corresponding beam simulation results in the RFQ are compared in Table II. We can also see a difference of the distribution between Figs. 34 and 28 , which are corresponding to currents of 1.6 and $0.1 \mathrm{emA}$, respectively. Although with two-harmonic prebunching, the beam transmission efficiency in the RFQ is slightly larger than that with three-harmonic bunching, indicating more particles are captured for acceleration. Figure 34 gives the explanation: for the three-harmonic case it is a waste of rf bucket. Besides, the output longitudinal emittance does not get worse because the rf bucket area of the RFQ is fixed [37]. The optimal mixture of the harmonics for the bunchers to maximize the RFQ

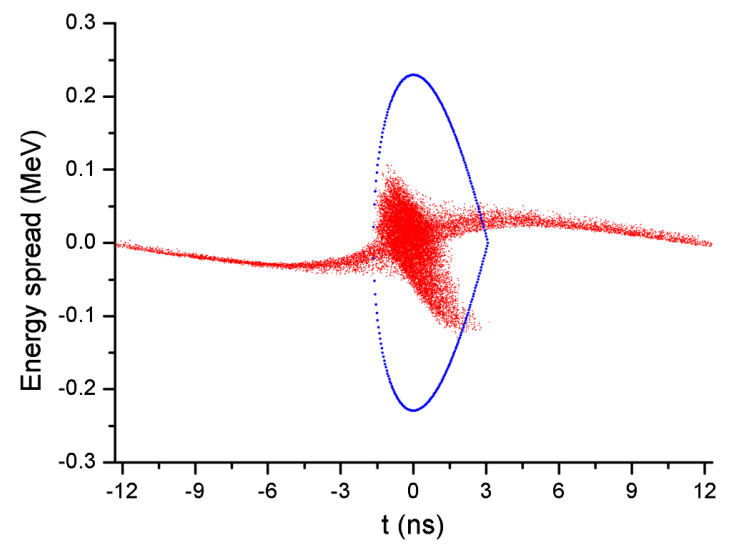

FIG. 33. Initial separatrix of the longitudinal motion and the simulated longitudinal distribution with space charge effect on. 

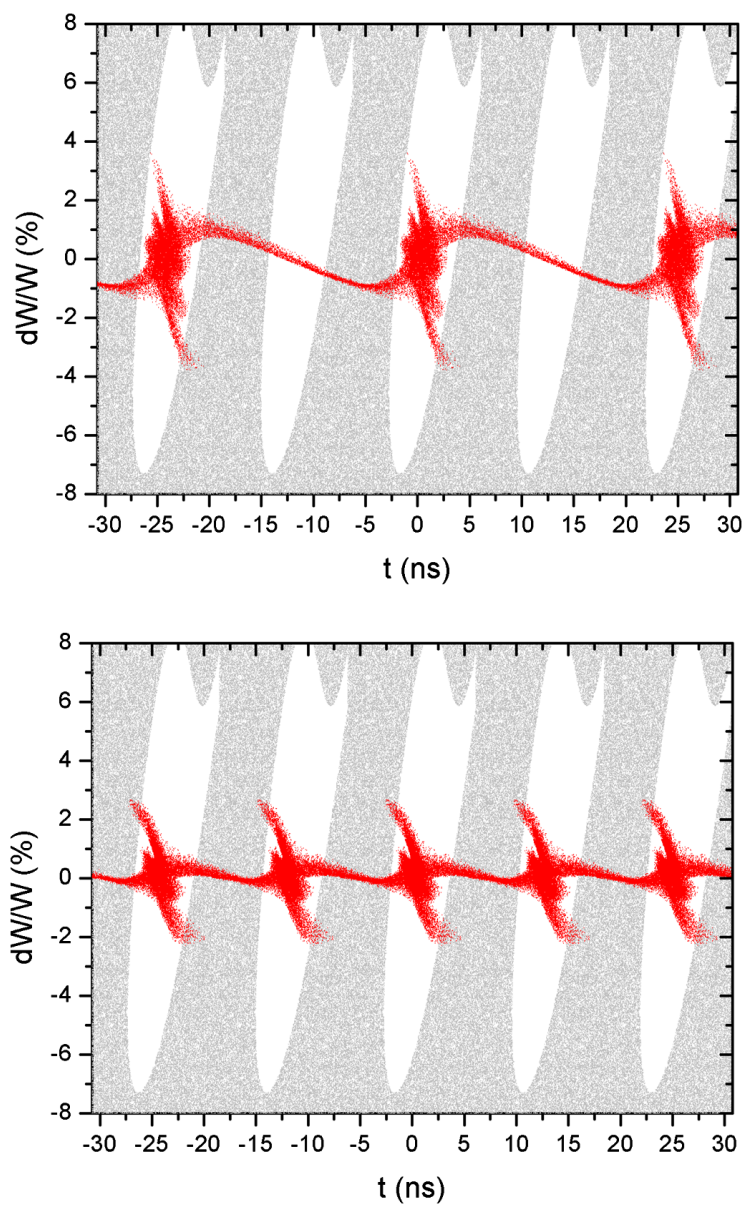

FIG. 34. Simulated beam longitudinal distributions after being bunched by a three-harmonic buncher (up) and a two-harmonic buncher (down) for a $1.6 \mathrm{emA} \mathrm{U}^{35+}$ beam.

transmission is shown in Table III. Owing to higher fundamental frequency of the two-harmonic buncher, the voltage amplitudes of the harmonics should be higher due to lower TTF. Therefore, a good cooling in the resonators should be considered.

TABLE II. Comparison of beam simulation results between using three-harmonic and two-harmonic prebunchers.

\begin{tabular}{lcc}
\hline \hline & $\begin{array}{c}\text { Three- } \\
\text { harmonic } \\
\text { buncher }\end{array}$ & $\begin{array}{c}\text { Two- } \\
\text { harmonic } \\
\text { buncher }\end{array}$ \\
\hline Objective ion & $\mathrm{U}^{35+}$ & $\mathrm{U}^{35+}$ \\
Beam current (emA) & 1.6 & 1.6 \\
$\begin{array}{l}\text { Transmission (accelerated) } \\
\text { Output transverse rms emittance } \\
\quad \varepsilon_{x} / \varepsilon_{y}(\pi \text { mm mrad) }\end{array}$ & $0.16 / 0.15$ & $0.16 / 0.15$ \\
$\begin{array}{l}\text { Output longitudinal rms emittance } \\
\varepsilon_{z, r m s}(\mathrm{keV} / \mathrm{u} \text { ns) }\end{array}$ & 0.30 & 0.30 \\
$\begin{array}{c}\text { Output longitudinal 99.9\% } \\
\text { emittance } \varepsilon_{z, 99.9 \%}(\mathrm{keV} / \mathrm{u} \mathrm{ns})\end{array}$ & 6.07 & 5.88 \\
\hline \hline
\end{tabular}

TABLE III. Voltage amplitudes of different harmonics calculated for the $\mathrm{U}^{35+}$ beam.

\begin{tabular}{lcc}
\hline \hline $\begin{array}{l}\text { Frequency } \\
(\mathrm{MHz})\end{array}$ & $\begin{array}{c}\text { Three-harmonic } \\
\text { buncher }\end{array}$ & $\begin{array}{c}\text { Two-harmonic } \\
\text { buncher }\end{array}$ \\
\hline 40.625 & $3192 \mathrm{~V}$ & $\ldots$ \\
81.25 & $2260 \mathrm{~V}$ & $4000 \mathrm{~V}$ \\
121.875 & $2027 \mathrm{~V}$ & $\ldots$ \\
162.5 & $\cdots$ & $9400 \mathrm{~V}$ \\
\hline \hline
\end{tabular}

\section{CONCLUSIONS}

An overall design of HIAF front end for transmission and acceleration of high-intensity highly charged heavy ions, such as $2 \mathrm{emA}^{238} \mathrm{U}^{35^{+}}$, has been conducted. Considering the particularity of the beam quality from an ECR ion source, beam extraction from the source has been modeled to obtain a more realistic particle distribution for further particle tracking simulation. The reliability of the extraction simulation was further verified by measurements. Transverse coupling is a typical property of the beam from ECR. Based on both simulation and experiment, two solenoids were arranged after the source for beam emittance exchange, which helps to reduce the beam emittance. Space charge compensation is a key issue in the LEBT design. A series of experiments, combining with the simulations, show that in the ECR CSS lines the mean space charge compensation could reach a high level, no less than $70 \%$, and the beam quality is mainly determined by the ion source tuning and plasma conditions.

The LEBT lines were designed as achromatic systems to avoid energy spread induced emittance growth. The multiparticle tracking simulation in the LEBT suggested a collimation system composed of three successive apertures can effectively eliminate the beam tails which account for $20 \%$ of the beam but attribute to about $70 \%$ of the emittance.

The design of HIAF RFQ will follow the development of LEAF-RFQ. The successful full-rf power conditioning and cw beam commissioning have proven an excellent design of LEAF-RFQ. Considering the longitudinal phase space distortions induced by the space charge effect for highintensity beams, the prebuncher has been redesigned to contain two harmonics with the fundamental frequency equal to the frequency of the RFQ to eliminate the satellite bunches. Beam simulation indicated that the two-harmonic buncher did not degrade the RFQ transmission efficiency and output beam quality, but required more rf power.

\section{ACKNOWLEDGMENTS}

The authors would like to thank B. Mustapha in ANL for the helpful discussion. This work is supported by the National Nature Science Foundation of China (Contracts No. 11427904 and No. 11575265). 
[1] J.C. Yang, in Proceedings of the 14th International Conference on Heavy Ion Accelerator Technology, HIAT-2018, Lanzhou, China, 2018 (IMP, Lanzhou, China, 2018), MOXBA01.

[2] J.C. Yang et al., High Intensity heavy ion Accelerator Facility (HIAF) in China, Nucl. Instrum. Methods Phys. Res., Sect. B 317, 263 (2013).

[3] H. W. Zhao, L. T. Sun, J. W. Guo, W. Lu, D. Z. Xie, D. Hitz, X. Z. Zhang, and Y. Yang, Intense highly charged ion beam production and operation with a superconducting electron cyclotron resonance ion source, Phys. Rev. Accel. Beams 20, 094801 (2017).

[4] H. W. Zhao, L. T. Sun, J. W. Guo, W. H. Zhang, W. Lu, W. Wu, B. M. Wu, G. Sabbi, M. Juchno, A. Hafalia, E. Ravaioli, and D.Z. Xie, Superconducting ECR ion source: From 24-28 GHz SECRAL to $45 \mathrm{GHz}$ fourth generation ECR, Rev. Sci. Instrum. 89, 052301 (2018).

[5] J. L. Biarrotte, P. Bertrand, and D. Uriot, in Proceedings of the 10th European Particle Accelerator Conference, EPAC-2006, Edinburgh, Scotland, 2006 (JACoW, Edinburgh, Scotland, 2006), WEPCH007.

[6] L. T. Sun, D. Leitner, G. Machicoane, E. Pozdeyev, V. Smirnov, S. B. Vorozhtsov, D. Winklehner, and Q. Zhao, Low energy beam transport for facility for rare isotope beams driver linear particle accelerator, Rev. Sci. Instrum. 83, 02 B705 (2012).

[7] X. Fang et al., in Proceedings of the 14th International Conference on Heavy Ion Accelerator Technology, HIAT2018, Lanzhou, China, 2018 (IMP, Lanzhou, China, 2018), MOPB15.

[8] Y. Yang et al., in Proceedings of the 5th International Particle Accelerator Conference, IPAC-2014, Dresden, Germany, 2014 (JACoW, Dresden, Germany, 2014), TUPRO054.

[9] T. Kalvas, O. Tarvainen, T. Ropponen, O. Steczkiewicz, J. Ärje, and H. Clark, IBSIMU: A three-dimensional simulation software for charged particle optics, Rev. Sci. Instrum. 81, 02B703 (2010).

[10] V. Toivanen, G. Bellodi, D. Küchler, A. Lombardi, R. Scrivens, and J. Stafford-Haworth, in Proceedings of the 21 st International Workshop on ECR Ion Sources, ECRIS2014, Nizhny Novgorod, Russia, 2014 (JACoW, Nizhny Novgorod, Russia, 2014), MOOAMH01.

[11] V. Toivanen, T. Kalvas, H. Koivisto, J. Komppula, and O. Tarvainen, Double einzel lens extraction for the JYFL 14 $\mathrm{GHz}$ ECR ion source designed with IBSimu, J. Instrum. 8, P05003 (2013).

[12] E. Pozdeyev, N. Bultman, G. Machicoane, G. Morgan, X. Rao, and Q. Zhao, in Proceedings of the 2013 Particle Accelerator Conference, PAC-2013, Pasadena, CA, 2013 (JACoW, Pasadena, CA, 2013), WEOAB1.

[13] C. Lyneis, D. Leitner, M. Leitner, C. Taylor, and S. Abbott, The third generation superconducting $28 \mathrm{GHz}$ electron cyclotron resonance ion source VENUS (invited), Rev. Sci. Instrum. 81, 02A201 (2010).

[14] P. Spädtke, K. Tinschert, R. Lang, J. Mäder, and J. Roßbach, Prospects of ion beam extraction and transport simulations (invited), Rev. Sci. Instrum. 79, $02 \mathrm{~B} 716$ (2008).
[15] M. A. Leitner, D. C. Wutte, and C. M. Lyneis, in Proceedings of the 2001 Particle Accelerator Conference, PAC-2001, Chicago, 2001 (JACoW, Chicago, 2001), MOPB004.

[16] Y. Yang, Y. J. Yuan, L. T. Sun, Y. C. Feng, X. Fang, Y. Cao, W. Lu, X. Z. Zhang, and H. W. Zhao, Transverse coupling property of beam from ECR ion sources, Rev. Sci. Instrum. 85, 113305 (2014).

[17] Y. Yang, Y. Liu, L. T. Sun, Y. J. Yuan, and H. W. Zhao, in Proceedings of the 22nd International Workshop on ECR Ion Sources, ECRIS-2016, Busan, Korea, 2016 (JACoW, Busan, Korea, 2016), WECO03.

[18] L. Groening, Concept for controlled transverse emittance transfer within a linac ion beam, Phys. Rev. ST Accel. Beams 14, 064201 (2011).

[19] P. Bertrand, J. L. Biarrotte, and D. Uriot, in Proceedings of the 10th European Particle Accelerator Conference, EPAC-2006, Edinburgh, Scotland, 2006 (JACoW, Edinburgh, Scotland, 2006), TUPLS081.

[20] X. H. Zhang, Y. J. Yuan, X. J. Yin, C. Qian, L. T. Sun, H. Du, Z. S. Li, J. Qiao, K. D. Wang, H. W. Zhao, and J. W. Xia, Beam dynamics of mixed high intensity highly charged ion Beams in the Q/A selector, Nucl. Instrum. Methods Phys. Res., Sect. A 857, 106 (2017).

[21] The beam dynamics code TRACK, www.phy.anl.gov/atlas/ TRACK.

[22] D. Winklehner, D. Leitner, D. Cole, G. Machicoane, and L. Tobos, Space-charge compensation measurements in electron cyclotron resonance ion source low energy beam transport lines with a retarding field analyzer, Rev. Sci. Instrum. 85, $02 \mathrm{~A} 739$ (2014).

[23] I. Soloshenko, Physics of ion beam plasma and problems of intensive ion beam transportation (invited), Rev. Sci. Instrum. 67, 1646 (1996).

[24] TraceWin code, http://irfu.cea.fr/dacm/logiciels/index4.php.

[25] G. Machicoane, N. Bultman, G. Morgan, E. Pozdeyev, X. Rao, J. Benitez, C. Lyneis, and L. T. Sun, in Proceedings of the 20th International Workshop on ECR Ion Sources, ECRIS-2012, Sydney, Australia, 2012 (JACoW, Sydney, Australia, 2012), THYO03.

[26] Y. Yang et al., in Proceedings of the 14th International Conference on Heavy Ion Accelerator Technology, HIAT2018, Lanzhou, China, 2018 (IMP, Lanzhou, China, 2018), MOPU05.

[27] W. Ma, L. Lu, X. Xu, L. Sun, Z. Zhang, W. Dou, C. Li, L. Shi, Y. He, and H. Zhao, Design of an $81.25 \mathrm{MHz}$ continuous-wave radio-frequency quadrupole accelerator for Low Energy Accelerator Facility, Nucl. Instrum. Methods Phys. Res., Sect. A 847, 130 (2017).

[28] W. Ma, L. Lu, T. Liu, L. B. Shi et al., Frequency and fields tuning of a heavy ion radio-frequency quadrupole accelerator, Nucl. Instrum. Methods Phys. Res., Sect. A 901, 180 (2018).

[29] J. Holzbauer, W. Hartung, F. Marti, E. Pozdeyev, and Q. Zhao, in Proceedings of the 2011 Particle Accelerator Conference, PAC-2011, New York, 2011 (JACoW, New York, 2011), TUP091.

[30] Q. Zhao, V. Andreev, M. Doleans et al., in Proceedings of the 22nd Linear Accelerator Conference, LINAC-2004, 
Lübeck, Germany, 2004 (DESY and GSI, Lübeck, Germany, 2004), THP03.

[31] P. N. Ostroumov, V. N. Aseev, A. Barcikowski, B. Clifft, R. Pardo, S. I. Sharamentov, and M. Sengupta, in Proceedings of 2007 Particle Accelerator Conference, PAC-2007, Albuquerque, New Mexico, USA, 2007 (JACoW, New York, 2007), WEPMN091.

[32] Y. Yang et al., in Proceedings of the 29th Linear Accelerator Conference, LINAC-2018, Beijin, China, 2018 (IHEP, Beijin, China, 2018), TUPO005.

[33] R. Ferdinand, P. Bertrand, M. Di Giacomo, H. Franberg, O. Kamalou, J. M. Lagniel, G. Normand, A. Savalle, F. Varenne, D. Uriot, and J. L. Biarrotte, in Proceedings of the 28th Linear Accelerator Conference, LINAC-2016, East
Lansing, MI, 2016 (JACoW, East Lansing, MI, 2016), WE1A06.

[34] CST Microwave Studio, http://www.cst.com/.

[35] C. Bontoiu, J. A. Duenas, J. Labrador, and I. Martel, in Proceedings of the 5th International Particle Accelerator Conference, IPAC-2014, Dresden, Germany, 2014 (JACoW, Dresden, Germany, 2014), MOPME059.

[36] R. E. Laxdal, Z. T. Ang, T. Au, S. Kiy, S. Rädel, O. Shelbaya, and V. Zvyagintsev, in Proceedings of the 29th Linear Accelerator Conference, LINAC-2018, Beijin, China, 2018 (IHEP, Beijin, China, 2018), MOPO092.

[37] S. Koscielniak, in Proceedings of the 1994 Linear Accelerator Conference, LINAC-1994, Tsukuba, Japan, 1994 (KEK, Tsukuba, Japan, 1994), pp. 526-528. 